\title{
A Statistical Approach to Cost-Sensitive AdaBoost for Imbalanced Data Classification
}

\author{
Honghan Bei $\mathbb{D}^{1,2}$ Yajie Wang ${ }^{1},{ }^{1}$ Zhaonuo Ren ${ }^{1},{ }^{1}$ Shuo Jiang $\mathbb{D}^{3},{ }^{3}$ Keran Li $\mathbb{D}^{2}{ }^{2}$ \\ and Wenyang Wang (D) 1,2 \\ ${ }^{1}$ School of Maritime Economics and Management, Dalian Maritime University, Dalian 116026, China \\ ${ }^{2}$ Collaborative Innovation Center for Transport Studies, Dalian Maritime University, Dalian 116026, China \\ ${ }^{3}$ School of Maritime Economics and Management, Dalian University of Technology, Dalian 116026, China
}

Correspondence should be addressed to Keran Li; likeran@dlmu.edu.cn and Wenyang Wang; wangwenyang@dlmu.edu.cn

Received 14 August 2021; Revised 13 September 2021; Accepted 24 September 2021; Published 23 October 2021

Academic Editor: Georgios Dounias

Copyright (c) 2021 Honghan Bei et al. This is an open access article distributed under the Creative Commons Attribution License, which permits unrestricted use, distribution, and reproduction in any medium, provided the original work is properly cited.

To address the imbalanced data problem in classification, the studies of the combination of AdaBoost, short for "Adaptive Boosting," and cost-sensitive learning have shown convincing results in the literature. The cost-sensitive AdaBoost algorithms are practical since the "boosting" property in AdaBoost can iteratively enhance the small class of the cost-sensitive learning to solve the imbalanced data issue. However, the most available cost-sensitive AdaBoost algorithms are heuristic approaches, which are improved from the standard AdaBoost algorithm by cost-sensitively adjusting the voting weight parameters of weak classifiers or the sample updating weight parameters without strict theoretic proof. The algorithms are appended the cost-sensitive factors to focus on the high-cost and small-class samples, but they have no procedures to show the best place to add the cost factors and the cost factor value set. To complete the cost-sensitive AdaBoost algorithms' framework, the present article has two main contributions. First, we summarize the popular cost-sensitive boosting algorithms in the literature and propose a generally comprehensive form. We name our specific one, the "AdaImC algorithm," which is typically appliable to solve the imbalanced data classification problem with theoretic proof. Second, a statistical approach to prove the AdaImC algorithm is proposed to verify the inner relationship between the cost parameters. We show that our proposed algorithm in the machine learning field is identical to the Product of Experts (PoE) model in the statistics field. Besides, a way to determine the cost parameter value by the statistical analysis is introduced. Several numeric studies are listed finally to support our proposed algorithm.

\section{Introduction}

Classification is one of the most important tasks in the machine learning and statistical field. Let $\mathbf{S}=\left\{\left(\mathbf{x}_{1}, y_{1}\right)\right.$, $\left.\left(\mathbf{x}_{2}, y_{2}\right), \ldots,\left(\mathbf{x}_{n}, y_{n}\right)\right\}$ be a binary training dataset for a classification problem, where $\mathbf{x}_{i} \in \mathbf{X} \subseteq \mathbf{R}^{l}$, in which $\mathbf{X}$ is a subset of $l$-dimensional vector space, and the response $y_{i} \in\{-1,1\}$ indicating two classes. Define $\mathbf{S}^{+}=\{(\mathbf{x}, y)$ $\in \mathbf{S}: y=1\}$ is the positive or minority class and $\mathbf{S}^{-}=\{(\mathbf{x}, y) \in \mathbf{S}: y=-1\}$ is the negative or majority class. The class types \{minority, majority\} and \{positive, negative\} are exchangeably described by $\mathbf{S}^{+}$and $\mathbf{S}^{-}$in this article when we talk about the imbalanced data problem. $\mathbf{S}^{+}$is the small class, but it is the interest of our research. Let $|\mathbf{A}|$ denote the number of the elements in a set $\mathbf{A}$. Define $N_{p}=\left|\mathbf{S}^{+}\right|$and $N_{n}=\left|\mathbf{S}^{-}\right|$, the number of samples in positive and negative class, respectively.

In classification, the imbalanced data problem is the most challenging one. The imbalanced class situation frequently appears in many fields, such as financial fraud detection [1], text recognition [2], sentiment classification [3], web classification [4], medical research [5], and oil exploration [6]. Under a balanced situation, $N_{n} \approx N_{p}$, many algorithms are available for classification, such as linear classifiers including logistic regression and naive Bayes classifier, support vector machines, decision trees, boosted trees, and random forest [7]. Although the above algorithms still could obtain an effective global accuracy rate in the 
imbalanced situation, the classification accuracy rate of $\mathbf{S}+$ could be low, which is the most interesting class. Typically, if $N_{n} \gg N_{p}$, this imbalanced condition in the different classes is called the imbalanced data problem. There is no strict definition for imbalanced data since some other influential factors such as small sample size and separability [8] would make the data "imbalanced" except the skewed distribution in the data. This vague definition requires that we not just focus on the data skewness if we are designing an imbalanced data classification algorithm and some comprehensive solutions to eliminate the imbalanced impact needed to be involved in the algorithm. In this paper, only the binary imbalanced class problem is studied. Multiple-class problems can be solved based on the oneversus-all scheme [9].

Two approaches $[10,11]$ to the class imbalanced problems, data-level and algorithm-level methods, are typically used for classification. The former is a preprocessing data method [12-14]. The basic idea is to preprocess the data to eliminate the imbalanced situation before the training process. On the other hand, the algorithm-level methods focus on training the classifier directly with the raw data. This approach mainly includes cost-sensitive learning [15] and ensemble schemes.

Cost-sensitive learning is a kind of object-oriented method. The common phenomenon is that many algorithms do not solve a real problem, although they have good theoretical properties. The reason is that external factors such as the cost of misclassification, the benefit of correct classification, and the efficiency substantially impact an algorithm's performance in the specific problems. At the present stage, most researchers in the machine learning field focus on designing and improving the structure of algorithms and ignore the impact of the external factor for an algorithm in the application. Typically, adjusting the existing algorithms by adding the external factor and importing the new evaluation measures based on the external factor improves the algorithms to make them more applicable in real problems. The cost-sensitive learning method considers the external factor by bringing in the different misclassification costs in the different classes to adjust the existing algorithms. For an imbalanced problem, by setting a higher cost for the misclassification in $\mathbf{S}^{+}$to force the classifier to skew to $\mathbf{S}^{+}$, the accuracy rate of $\mathbf{S}^{+}$would obtain improvement. Broadly, cost-sensitive learning is categorized into two. The first is to design the classifiers directly with cost-sensitive parameters, which is called the direct method. The most famous ones are ICET [16] and cost-sensitive decision trees [17]. The other is to design a classifier that converts any existing cost-insensitive classifiers into cost-sensitive ones, which is called the cost-sensitive metalearning method. It can be further categorized into thresholding and sampling. The famous thresholding methods are MetaCost [18], cost-sensitive naive Bayes [19], and empirical thresholding [17]. The famous sampling cost-sensitive metalearning methods are Costing [20] and Weighting [21]. The above seven traditional cost-sensitive classification algorithms are conducted in the numeric section so as to compare with the proposed ones.
Ensemble schemes include boosting [22-24] and bagging [25]. The boosting method is based on the theorem of Equivalence of strong and weak learnability [26]. The AdaBoost algorithm is one of the most popular boosting methods. AdaBoost, the abbreviation of Adaptive Boosting, is a machine learning algorithm formulated by Yoav Freund and Robert Schapire [27]. AdaBoost combines a series of weak classifiers and builds the final strong one to enhance the final classifier's performance. When the weak classifiers are boosted, they have the vote weights related to every weak classifier's accuracy rate. After a weak classifier is added, the data weight for the next weak classifier's training is also readjusted to give a higher weight for the misclassified instances. Thus, future weak classifiers focus on the instances that previous weak classifiers misclassified.

Cost-sensitive learning and AdaBoost methods are both powerful and efficient for data classification. The combination of cost-sensitive learning and AdaBoost should provide a more potent solution. We call this kind of method "cost-sensitive AdaBoost." The basic idea is to add a cost factor to each of the weak classifiers and then boost them to build the final classifier based on the boosting method in the AdaBoost algorithm. The cost factor used here comes from the empirical rule in the specific problem and makes the algorithms more applicable if we import the cost-related evaluation measure. The cost factor can also help every weak classifier focus on the high-cost class to eliminate the imbalance. The cost-sensitive AdaBoost can make the algorithms more applicable by considering the cost of misclassification and improve the classification accuracy rate of $\mathbf{S}^{+}$by adding high cost to the minority class [28].

The structure of this paper is as follows. Section 2 reviews the AdaBoost and cost-sensitive learning algorithms. The combinations of these two algorithms in the literature are stated, and a comprehensively general form is proposed. In Section 3, we proposed our model, AdaImC Algorithm, with the theoretic proofs. A statistical approach to the AdaImC algorithm is given in Section 4. The inner relationship between the parameters in our model is shown. The results of the numeric studies with the simulated datasets and real datasets are listed in Section 5.

\section{AdaBoost and Cost-Sensitive Learning Algorithms}

2.1. Classical AdaBoost. A review of the classical AdaBoost is stated in Algorithm 1.

A weak classifier $h_{t}(\mathbf{x})$ presents a map from $\mathbf{X}$ to $\mathbf{R}$, and $\alpha_{t}$ is the corresponding vote weight parameter. Note that $\alpha_{t}$ in Step 2 is chosen based on minimizing the exponential loss function [29], which implies that the more accurate a weak classifier is, the larger vote weight it has. Step 3 presents the data weight, $D_{t}(i)$. If instant $\left(\mathbf{x}_{i}, y_{i}\right)$ is misclassified by $h_{t}(\mathbf{x})$, then $D_{t+1}(i)>D_{t}(i)$ in $h_{t+1}(\mathbf{x})$. On the other hand, if instant $\left(\mathbf{x}_{i}, y_{i}\right)$ is classified correctly by $h_{t}(\mathbf{x})$, then $D_{t+1}(i) \leq D_{t}(i)$ in $h_{t+1}(\mathbf{x})$ inversely [30]. The following is shown in [29].

Lemma 1. When $h_{t}(\mathbf{x})$ has a range of $[-1,1]$,

$$
\frac{1}{n}\left|i: h\left(\mathbf{x}_{i}\right) \neq y_{i}\right| \leq \prod_{t} \mathbf{Z}_{t}
$$


Input: the training data: $\left(\mathbf{x}_{1}, y_{1}\right),\left(\mathbf{x}_{2}, y_{2}\right), \ldots,\left(\mathbf{x}_{n}, y_{n}\right)$.

(0) Choose the number of weak classifiers $T$, let $t=1$, and initialize the sample weight $D_{t}(i)=1 / n, i=1, \ldots, n$;

(1) Under $D_{t}(i)$, find the weak classifier $h_{t}(\mathbf{x}) \in\{-1,1\}$ minimizing the weighted error rate $\epsilon_{t}=\sum_{h_{t}\left(\mathbf{x}_{i}\right) \neq y_{i}} D_{t}(i)$;

(2) Calculate the weak classifier weight: $\alpha_{t}=(1 / 2) \ln \left\{\left(1-\epsilon_{t}\right) / \epsilon_{t}\right\}$;

(3) Update $D_{t+1}(i)=D_{t}(i) \exp \left\{-\alpha_{t} y_{i} h_{t}\left(\mathbf{x}_{i}\right)\right\} / \sum_{j=1}^{n} D_{t+1}(j), i=1,2, \ldots, n$;

(4) If $t=T$, stop the iteration, else let $t=t+1$ and return to Step 1.

Output: the final strong classifier is $H(\mathbf{x})=\operatorname{sign}\left\{\sum_{t=1}^{T} \alpha_{t} h_{t}(\mathbf{x})\right\}$.

Algorithm 1: The classical AdaBoost [27, 29].

where $|a|$ is an indicator, $|a|=1$ if $a$ is true and $|a|=0$ if $a$ is false, and $\mathbf{Z}_{t}=\sum_{i=1}^{n} D_{t}(i) \exp \left\{-\alpha_{t} y_{i} h_{t}\left(\mathbf{x}_{i}\right)\right\}, t=1,2, \ldots, T$.

The left formula of (1) is the empirical error rate, and the right formula is the corresponding upper boundary. Minimizing $\mathbf{Z}_{t}$ on each iteration to decrease the error rate, we obtain

$$
\alpha_{t}=\frac{1}{2} \ln \left\{\frac{1-\epsilon_{t}}{\epsilon_{t}}\right\},
$$

where $\epsilon_{t}=\sum_{h_{t}\left(\mathbf{x}_{i}\right) \neq y_{i}} D_{t}(i)$. The training error should be less than 0.5 , the random guessing error rate, that is,

$$
\sum_{h_{t}\left(\mathbf{x}_{i}\right)=y_{i}} D_{t}(i)>\sum_{h_{t}\left(\mathbf{x}_{i}\right) \neq y_{i}} D_{t}(i) .
$$

This also induces that all $\alpha_{t}$ should be positive.

Exponential Loss Function is used in AdaBoost to produce $D_{t}(i)$. The Exponential Loss Function is defined as

$$
L(y, f(\mathbf{x}))=\exp (-y f(\mathbf{x})),
$$

where

$$
f(\mathbf{x})=\sum_{t=1}^{T} \alpha_{t} h_{t}(\mathbf{x}) .
$$

On each iteration of training the weak classifier, one must solve

$$
\begin{aligned}
\left(\alpha_{t}, h_{t}\right) & =\arg \min _{\alpha_{t}, h_{t}} \sum_{i=1}^{n} \exp \left\{-y_{i}\left(f_{t-1}\left(\mathbf{x}_{i}\right)+\alpha_{t} h\left(\mathbf{x}_{i}\right)\right)\right\} \\
& =\arg \min _{\alpha_{t}, h_{t}} \sum_{i=1}^{n} D_{t}(i) \exp \left\{-\alpha_{t} y_{i} h\left(\mathbf{x}_{i}\right)\right\}
\end{aligned}
$$

where $D_{t}(i)=\exp \left(-y_{i} f_{t-1}\left(\mathbf{x}_{i}\right)\right)$. For any fixed positive value of $\alpha_{t}$, (6) can be rewritten as

$$
\begin{aligned}
h_{t} & =\arg \min _{h_{t}} \sum_{i: y_{i} \neq h_{t}\left(\mathbf{x}_{i}\right)}^{n} D_{t}(i) \\
& =\arg \min _{h_{t}} \epsilon_{t} .
\end{aligned}
$$

When $h_{t}(x)$ is determined by (7), $\alpha_{t}$ can be fixed in (2):

$$
D_{t+1}(i) \propto D_{t}(i) \exp \left\{-\alpha_{t} y_{i} h_{t}\left(\mathbf{x}_{i}\right)\right\}, \quad i=1,2, \ldots, n .
$$

2.2. Cost-Sensitive Learning. Cost-sensitive learning is an essential method in the algorithms approach to solving the imbalanced data classification problem. Cost-sensitive learning brings different costs for different classes when misclassification happens $[17,31,32]$. It is more "needs satisfying" in real problems. Generally, classification methods in the machine learning field focus on finding the classifier with a minimum error rate. However, the costsensitive learning method attempts to obtain a classifier with the minimum misclassified cost. The primary difference between the cost-sensitive learning method and other algorithms is the evaluation criteria. The confusion matrix is given in Table 1 .

The traditional cost-insensitive classifiers focus on minimizing the global error rate, $\epsilon=\mathrm{FN}+\mathrm{FP}$, on obtaining a higher classification accuracy rate, but there is no restriction or requirement for the percentage of FN and FP in $\epsilon$. From the cost-sensitive perspective, cost-insensitive classifiers assume that the cost factor of false-negative prediction and false-positive prediction is the same. In real problems, this assumption does not usually hold. For example, a bank would use machine learning algorithms to decide if accept a loan request. There are two evaluation criteria in this biclass classification problem:

(a) Classification result has the minimum misclassified error rate

(b) Classification result has the minimum misclassified cost

When all the false decisions have the same cost, (a) and (b) are equal. However, FP's cost, which does not accept qualified customers' requests, is lower than FN's cost that accepts nonqualified customers' requests. Table 2 shows a Cost Confusion Matrix describing the cost under the different situations in Table 1 . In cost-sensitive learning, we are seeking a classifier minimizing $c=\mathrm{FP} * c_{1,0}+\mathrm{FN} * c_{0,1}$. Cost-sensitive learning is practical for many other real problems, such as hazardous act detection and cancer diagnosis. The formal definition of Cost Confusion Matrix is as follows.

Definition 1. Let $\mathbf{C}_{m \times m}$ be the cost matrix for a given training data, where $m$ is the number of classes. Every nonnegative element $c_{i, j}$ in $\mathbf{C}_{m \times m}$ means the cost of predicting class $i$, but the true class is $j$, here $i, j=1,2,3, \ldots, m$. 
TABLE 1: Confusion matrix.

\begin{tabular}{|c|c|c|c|c|}
\hline & & \multicolumn{2}{|c|}{ Prediction } & \multirow{2}{*}{ Row total } \\
\hline & & Positive & Negative & \\
\hline \multirow{3}{*}{ Truth } & Positive & $\mathrm{TP}=$ true positive rate & $\mathrm{FN}=$ false negative rate & $P$ \\
\hline & Negative & $\mathrm{FP}=$ false positive rate & $\mathrm{TN}=$ true negative rate & $N$ \\
\hline & Column total & $P^{\prime}$ & $N^{\prime}$ & 1 \\
\hline
\end{tabular}

TABle 2: Cost confusion matrix.

\begin{tabular}{cccc}
\hline & & \multicolumn{2}{c}{ Prediction } \\
& & Positive & Negative \\
\hline \multirow{2}{*}{ Truth } & Positive & $c_{1,1}$ & $c_{0,1}$ \\
& Negative & $c_{1,0}$ & $c_{0,0}$ \\
\hline
\end{tabular}

Remark 1

(a) Cost confusion matrix needs to be determined before the training process. Most times, it is provided by the data collector based on the empirical rule.

(b) Cost confusion matrix needs to obey the reasonableness condition, which means that the cost of misclassification should be higher than the cost of correct classification, i.e., $c_{i, j}>c_{j, j}, i \neq j$ for $i, j=1,2, \ldots, m$. Usually, people treat $c_{j, j}=0$ and $c_{i, j}>0, i \neq j$ for simplification.

(c) In an imbalanced biclass problem, the positive class is the primary interest, so that the cost of FN should be higher than the cost of FP, i.e., $c_{\mathrm{FN}}>c_{\mathrm{FP}}$. By setting this cost, the algorithm focuses on the positive class in the training process.

Cost-sensitive learning is equivalent to the optimization problem applied to a specific loss function.

Theorem 1. Let $c_{i, j}$ defined in Definition 1 be the cost of predicting class $i$ when the true class is $j$. If $i=j$, the prediction is correct; if $i \neq j$, the prediction is incorrect, where $i, j=1,2,3, \ldots, m$. For a cost-sensitive learning algorithm $\mathbf{A}$, the optimal prediction for an instant in class $i$ is to minimize

$$
L(\mathbf{x}, i)=\sum_{j} P(j \mid \mathbf{x}) c_{i, j}
$$

where $(\mathbf{x}, i)$ means the prediction class of $\mathbf{x}$ is $i$ and $P(j \mid \mathbf{x})$ means the probability of $\mathbf{x}$ belongs to class $j$ under the algorithm A.

Corollary 1. In the biclass case, Theorem 1 can be written as

$$
\begin{aligned}
P(j & =-1 \mid \mathbf{x}) c_{1,-1}+P(j=-1 \mid \mathbf{x}) c_{1,1} \\
& \leq P(j=-1 \mid \mathbf{x}) c_{-1,-1}+P(j=-1 \mid \mathbf{x}) c_{-1,1},
\end{aligned}
$$

which is equivalent to

$$
(1-p) c_{1,-1}+p c_{1,1} \leq(1-p) c_{-1,-1}+p c_{-1,1}
$$

where $p=P(j=1 \mid \mathbf{x})$.
Corollary 2. Let the threshold to make the optimal decision is $p^{*}$, which has the property of

$$
\left(1-p^{*}\right) c_{1,-1}+p^{*} c_{1,1}=\left(1-p^{*}\right) c_{-1,-1}+p^{*} c_{-1,1},
$$

and we have

$$
p^{*}=\frac{c_{1,-1}-c_{-1,-1}}{c_{1,-1}-c_{-1,-1}+c_{-1,1}-c_{1,1}} \text {. }
$$

Under the reasonableness condition, the optimal prediction is the positive class (class 1 ) if and only if $p \geq p^{*}$.

The description of the general cost-sensitive learning algorithm is given in Algorithm 2.

Although some cost-sensitive learning methods are starting from building a new algorithm structure based on available cost information, such as the cost-sensitive decision tree pruning method [33], most of the existing costsensitive learning methods are adjusted from the cost-insensitive algorithms by adding the cost information [34]. This approach transfers the minimizing global error algorithm to the minimizing expected cost algorithm, and it is highly optimized for general use, including the rescaling method and reweighted method. Typically, the rescaling method is a resampling approach that preprocesses the training data based on the cost information to change the data distribution before the training process. The classifier will be biased to the cost-sensitive class and improve its classification accuracy rate. Rescaling is another data-level method but considering the cost-sensitive information [1, 35-37]. On the other hand, the reweighted approach, including the cost-sensitive ensemble learning method, focuses on adjusting the cost-insensitive algorithms. It brings cost information to obtain cost-sensitive property and then boosts a series of cost-sensitive weak classifiers to obtain a strong one. MetaCost [18] and Proportion-Cost [20] are two other interesting reweighted approaches in cost-sensitive learning. Figure 1 gives a family tree to illustrate the relationship between all the classifiers we mentioned above.

2.3. Cost-Sensitive AdaBoost. We see several kinds of costsensitive AdaBoost algorithms in the literature, such as AdaCost series algorithms [38], AdaC series algorithms [8], CBS series algorithms [39], and Asymmetric Boosting algorithm [40]. These algorithms are the most popular ones, but they are heuristic without strict theoretic proof. Their basic idea is to add cost factors to the vote weights of the weak classifiers $\alpha_{t}$ or the data weights $D_{t}$ in AdaBoost. Table 3 summarizes adjusting the classical AdaBoost to obtain the above existing cost-sensitive AdaBoost algorithms. 
Input: the training data: $\left(\mathbf{x}_{1}, y_{1}, c_{y_{1}}\right),\left(\mathbf{x}_{2}, y_{2}, c_{y_{2}}\right), \ldots,\left(\mathbf{x}_{n}, y_{n}, c_{y_{n}}\right)$. Every $\mathbf{x}_{i}$ is an $l$-dimensional variable in $\mathbf{X}, y_{i}$ is a class label in $\mathbf{Y}=\{-1,1\}$, and $c_{y_{i}} \subseteq[0,+\infty)$ is the associating misclassification cost for every class;

(1) Define the data weight $D$ based on $\mathbf{X} \times \mathbf{Y}$ and the cost matrix $C_{2 \times 2}$ based on Definition 1;

(2) Under this $D$ and $C_{2 \times 2}$, find the classifier $h_{t}(\mathbf{x})$ minimizing $E_{\mathbf{X}, \mathbf{Y} \sim D}[\operatorname{cost}(y, y h(\mathbf{x}))]$;

Output: the final classifier is $h(\mathbf{x})$.

Algorithm 2: The biclass cost-sensitive learning algorithm [8].

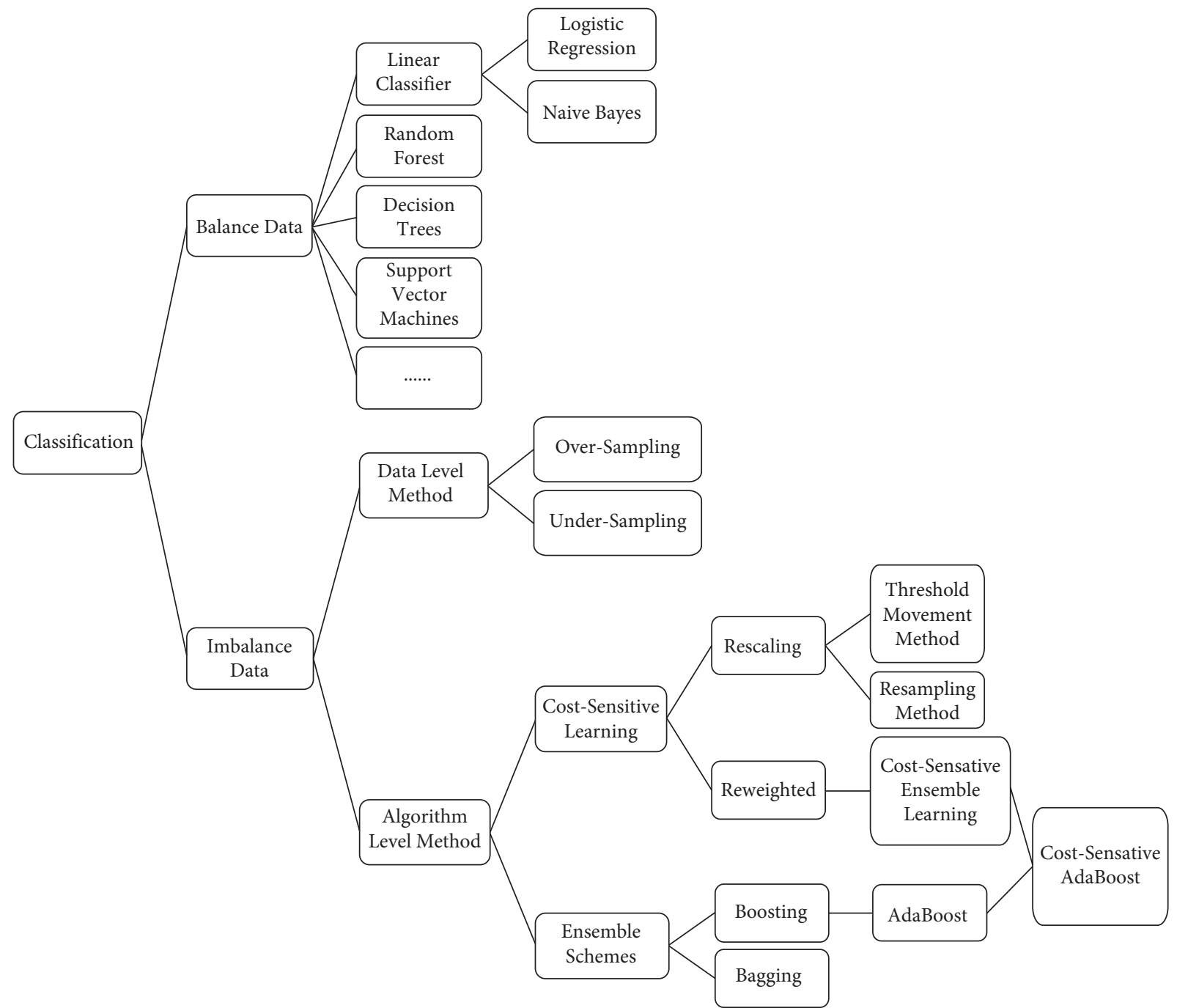

FIGURE 1: Various methods for classification.

Some other cost-sensitive AdaBoost algorithms, such as CGAda [41] and CSAda [42], are not widespread, which we do not consider in this article. Some researchers have done excellent work to analyze the existing cost-sensitive AdaBoost algorithms in Table 3, which are used frequently in the machine learning field. Cao et al. [14] listed the weakness of some popular cost-sensitive AdaBoost algorithms, including those in Table 3, and concluded that most of them are heuristic and lack the theoretical analysis. Nikolaou et al. [43] gave a summary of them and analyzed them from a mathematical perspective. One of the most critical properties of boosting is the robustness of the resulting, which means that the final classifier tends to continue reducing the test error even after perfect classification is reached on the training set. The heuristic adjustment of AdaBoost would lose this property in Boosting.

The adjusted point in the AdaCost is the cost function $\beta_{t}^{i}$. In the high-cost class, $\beta_{t}^{i}$ is low in the correctly classified situations and is high in the misclassified situations. Nevertheless, AdaCost did not consider the change in $\alpha_{t}$. In 


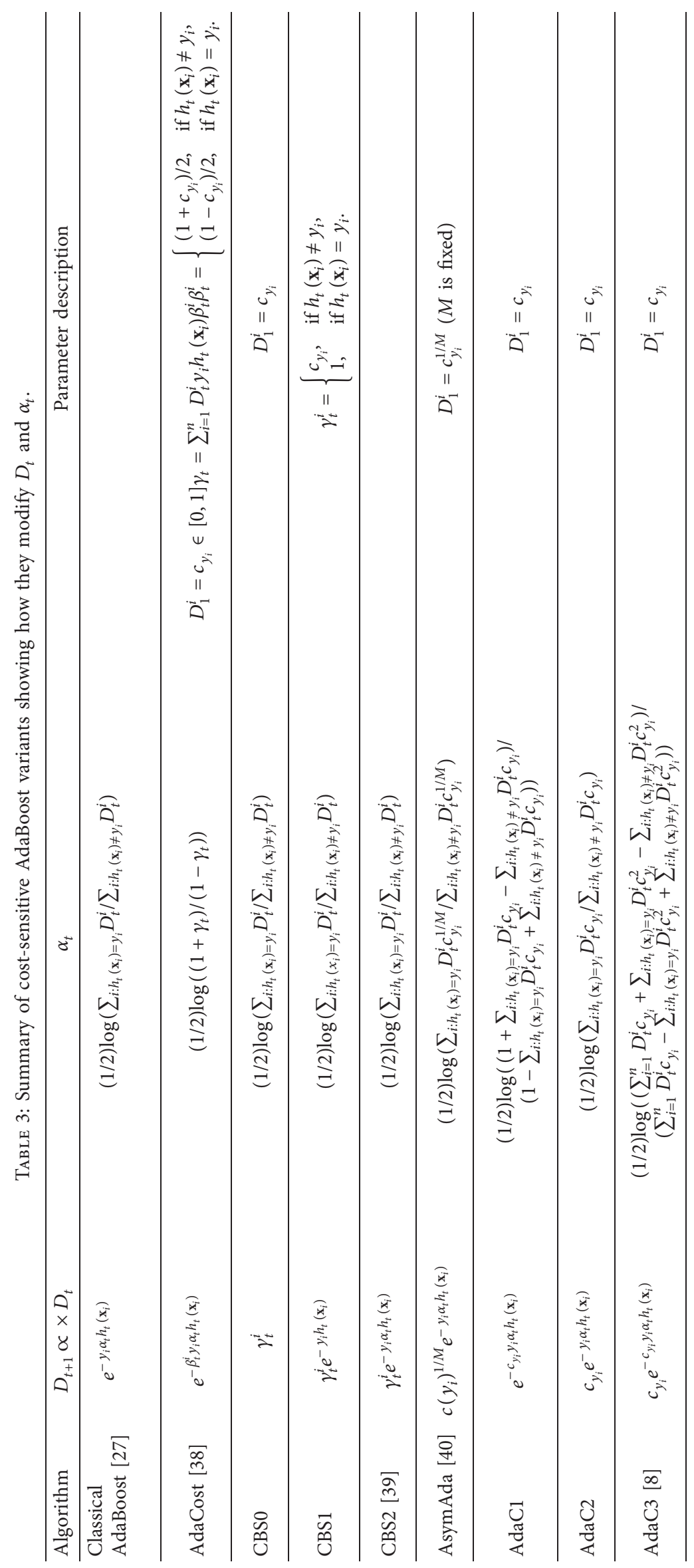


order to keep the reasonability of $\alpha_{t}\left(\alpha_{t}>0\right)$, every weak classifier needs to satisfy $\sum_{i=1}^{n} D^{i} \beta_{h\left(\mathbf{x}_{i}\right)=y_{i}}^{i}>\sum_{i=1}^{n} D^{i} \beta_{h\left(\mathbf{x}_{i}\right) \neq y_{i}}^{i}$. This restriction requires a very high accuracy rate for the weak classifiers. In an imbalanced data classification problem, people can often not obtain the powerful weak classifiers for boosting. Because of the limit of efficiency, AdaCost does not perform well in imbalanced data classification.

CSB is a further study based on AdaCost. Ting and Ming [39] compared CSB series algorithms and AdaCost algorithm to conclude that CSB performs better than AdaCost. However, the apparent shortcomings of CSB are that it only adjusts the data weight $D_{t}$ and does not adjust the corresponding weak classifier vote weight $\alpha_{t}$. This heuristic adjusting deviates from the original bboosting property in AdaBoost.

AsymAda, short for Asymmetric Boosting, applies the gradient descent optimization method to the cost-sensitive loss function $L(\mathbf{x}, y, h(\mathbf{x}))=E_{\mathbf{x}, y \sim D}\left[I(y=1) e^{-}\right.$ $\left.c_{y_{i}} y h(\mathbf{x})+I(y=-1) e^{-c_{y_{i}} y h(\mathbf{x})}\right]$. Nevertheless, people have to use a numerical method such as the bisection search to find $\alpha_{t}$ [42]. This would increase the complexity of algorithms and require more time to train the model.

AdaC series algorithms consider three different positions of the cost factor in $D_{t}$ and then infer $\alpha_{t}$ based on $D_{t}$. AdaC series algorithms have strict theoretical analysis to support the algorithm's reasonability following the proofs in [29]. The authors concluded that AC2 is superior to its rivals, and several numerical studies support this conclusion. Our proposed model starts with AdaC3. In AdaC3, $D_{t+1}^{i} \propto c_{y_{i}} e^{c_{y_{i}} y_{i} \alpha_{t} h_{t}\left(\mathbf{x}_{i}\right)} \times D_{t}^{i}$, two positions contain the same cost factor $c_{y_{i}}$. $c_{y_{i}}$ outside of the exponential is the adjustment for $D_{t}$, but $c_{y_{i}}$ inside of the exponential is the adjustment for $\alpha_{t}$. It is unnecessary to set the same cost factor value on these two positions. Based on this, the AdaC3 can be expanded to a generally comprehensive formation of cost-sensitive AdaBoost in the following.

Definition 2. Given the training data $\left(\mathbf{x}_{1}, y_{1}, c_{y_{1}}\right)$, $\left(\mathbf{x}_{2}, y_{2}, c_{y_{2}}\right), \ldots,\left(\mathbf{x}_{n}, y_{n}, c_{y_{n}}\right)$, where $\mathbf{x}_{i}$ is the feature variable, $y_{i}=\{-1,1\}$ is the class variable, and $c_{y_{i}}$ is the misclassified cost for every instant. Let $\mathbf{C}_{1}^{i}\left(c_{y_{i}}\right)$ is a function of $c_{y_{i}}$ and $\mathrm{C}_{2}^{t}\left(c_{y_{i}}, D_{t}^{i}\right)$ is a function of $c_{y_{i}}$ and $D_{t}^{i}$. The cost-sensitive adjustment of $D_{t}$ in AdaBoost is

$$
D_{t+1}^{i} \propto \mathbf{C}_{1}^{i}\left(c_{y_{i}}\right) e^{\mathrm{C}_{2}^{t}\left(c_{y_{i}}, D_{t}^{i}\right) y_{i} h_{t}\left(\mathbf{x}_{i}\right)} \times D_{t}^{i},
$$

This comprehensively general formation includes all the algorithms in Table 3. The general form of the cost-sensitive AdaBoost algorithm is given in Algorithm 3.

This article aims to find specific formations of $\mathbf{C}_{1}^{i}\left(c_{y_{i}}\right)$ and $\mathbf{C}_{2}^{t}\left(c_{y_{i}}, D_{t}^{i}\right)$ for designing a new cost-sensitive AdaBoost for imbalanced data classification. The proposed model is biased to the positive class by the cost information and obeys the boosting property.

2.4. Evaluation Measures. In the cost-sensitive learning method, the most popular evaluation measure is to compare the cost of different algorithms. Ting [39] used two costrelated measures to evaluate the performance of algorithms:

(1) The total cost of misclassification made by the classifier on the test data, i.e., $\sum_{i: y_{i} \neq h\left(\mathbf{x}_{i}\right)} c_{y_{i}}$

(2) The number of high-cost errors is the number of misclassified samples in the high-cost class

Other evaluation measures, such as the average misclassification cost for a series of cost values, are also used frequently. For example, let $c_{-1,1}=1$ and $c_{1,-1}=0.1,0.2,0.3,0.4,0.5,0.6,0.7,0.8,0.9,1$. The average misclassified cost for all these ten pairs of values is the evaluation measure.

Because our interest is the imbalanced data classification, some people only focus on the accuracy rate and use the traditional evaluation measures of classification for cost-sensitive AdaBoost such as F-1 Measure, Recall, and Precision [8]. This is reasonable when our interest is the accuracy rate of the positive class, and the cost is only a tool to neutralize the imbalanced situation. Cost-sensitive adjustment in AdaBoost is just a tool to force the algorithm to focus on the positive class. Based on Confusion Matrix 1, we can define some basic evaluation measures as follows:

$$
\begin{aligned}
& \text { Global Accuracy }=\mathrm{TP}+\mathrm{TN} \\
& \text { Sensitivity }=\mathrm{Recall}=\mathrm{TP} / P \\
& \text { Specificity }=\mathrm{TN} / N \\
& \text { Precision }=\mathrm{TP} / P^{\prime} \\
& F-1 \text { Measure }=(2 \times \text { Precision } \times \\
& \text { Sensitivity }) /(\text { Precision }+ \text { Sensitivity })
\end{aligned}
$$

Sensitivity(Recall) and Precision are the measures for accessing the classification performance of $\mathbf{S}^{+}$. $F-1$ Measure was suggested in [44] to integrate the Sensitive and Precision as an average, representing a harmonic mean between Sensitive and Precision. The harmonic mean of two numbers is closer to the smaller one. So, a high $F-1$ Measure can ensure that the Sensitive and Precision are both high.

This paper will consider the traditional evaluation measures for the real data studies and cost-related evaluation measures for the simulation data studies.

\section{AdaImC Algorithm}

The term AdaImC means the cost-sensitive AdaBoost algorithm designed for imbalanced data classification. Recall the general expression of the adjustment in cost-sensitive AdaBoost in Algorithm 3:

$$
D_{t+1}^{i} \propto \mathbf{C}_{1}^{i}\left(c_{y_{i}}\right) e^{\mathbf{C}_{2}^{t}\left(c_{y_{i}}, D_{t}^{i}\right) y_{i} h_{t}\left(\mathbf{x}_{i}\right)} \times D_{t}^{i},
$$

where $\mathbf{C}_{1}^{i}\left(c_{y_{i}}\right)$ works for adding the cost factor to the data weight update procedure and $\mathbf{C}_{2}^{t}\left(c_{y_{i}}, D_{t}^{i}\right)$ is an adjustment for $\alpha_{t}$.

Definition 3. The specific adjustment of $D_{t}$ in AdaImC is 
Input: the training data: $\left(\mathbf{x}_{1}, y_{1}, c_{y_{1}}\right),\left(\mathbf{x}_{2}, y_{2}, c_{y_{2}}\right), \ldots,\left(\mathbf{x}_{n}, y_{n}, c_{y_{n}}\right)$, where $\mathbf{x}_{i}$ is the feature variable, $y_{i} \in\{-1,1\}$ is the class variable, and $c_{y_{i}}$ is the misclassification cost for every instant.

(0) Choose the number of weak classifiers $T$, let $t=1$, and initialize the sample weight $D_{t}(i)=c_{y_{i}}, i=1, \ldots, n$;

(1) Under this $D_{t}(i)$, find the weak classifier $h_{t}(\mathbf{x}) \in\{-1,1\}$ minimizing the weighted error rate $\epsilon_{t}=\sum_{h_{t}}\left(\mathbf{x}_{i}\right) \neq y_{i} D_{t}(i)$;

(2) Choose $\alpha_{t} \in R^{+}$;

(3) Update $D_{t+1}^{i} \propto \mathbf{C}_{1}^{i}\left(c_{y_{i}}\right) e^{\mathbf{C}_{2}^{t}\left(c_{y_{i}}, D_{t}^{i}\right) y_{i} h_{t}\left(\mathbf{x}_{i}\right)} \times D_{t}^{i}, i=1,2, \ldots, n$;

(4) If $t=T$, stop the iteration, else let $t=t+1$ and return to Step 1.

Output: the final strong classifier is $H(\mathbf{x})=\operatorname{sign}\left\{\sum_{t=1}^{T} \alpha_{t} h_{t}(\mathbf{x})\right\}$.

Algorithm 3: The general cost-sensitive AdaBoost.

$$
D_{t+1}^{i} \propto D_{t}^{i} c_{1}^{\left(1-y_{i}\right) / 2} \exp \left[-y_{i} h_{t}\left(\mathbf{x}_{i}\right)\left(\alpha_{t}+c_{2}\right)\right]
$$

where $c_{1} \in(0,1)$ and $c_{2} \in(0,+\infty]$.

The adjustment in Definition 3 is similar to the AdaC3 algorithm. The main difference is that we add a new parameter $c_{2}$ to $\alpha_{t}$ instead of multiplying $c_{y_{i}}$ by $\alpha_{t} \cdot c_{1}^{\left(1-y_{i}\right) / 2}$ is the adjustment for the sample weight parameters. For the positive class, $y_{i}=1$ and $c_{1}^{\left(1-y_{i}\right) / 2}=1$; for the negative class, $y_{i}=-1$ and $c_{1}^{\left(1-y_{i}\right) / 2}=c_{1}$. This adjustment enhances the weight parameters for the positive class since $c_{1}<1$. The purpose of the adjustment $c_{2}$ is to add effect on the voting weight parameters $\alpha_{t}$ of the weak classifiers so that the outstanding weak classifiers can contribute more to the final classifier.

Remark 2. In Definition 3, $0<c_{1}<1$ and $c_{2}>0$.

The reason of $0<c_{1}<1$ is obvious because $c_{1}^{\left(1-y_{i}\right) / 2}=$ $\left\{\begin{array}{ll}1, & \text { if } y_{i}=1, \\ c_{1}, & \text { if } y_{i}=-1 .\end{array}\right.$ The positive class cost should be higher than the negative class, and all the cost values are nonnegative. $c_{2}>0$ gives a heavier effect to $\alpha_{t}$.

Theorem 2. Assuming Algorithm 1 replaces the equation in Algorithm 3, we have

$$
\frac{1}{n} \sum_{i=1}^{n}\left|h\left(\mathbf{x}_{i}\right) \neq y_{i}\right| \leq \frac{1}{c_{1}} \prod_{t=1}^{T} \mathbf{Z}_{t},
$$

where $|a|=1$ if $a$ is true and $|a|=0$ if $a$ is false, and

$$
\mathbf{Z}_{t}=\sum_{i=1}^{n} D_{t}^{i} c_{1}^{\left(1-y_{i}\right) / 2} \exp \left[-y_{i} h_{t}\left(\mathbf{x}_{i}\right)\left(\alpha_{t}+c_{2}\right)\right] .
$$

For the proof of Theorem 2, see Appendix A

Theorem 2 indicates an upper boundary of the global error rate for the AdaImC algorithm. The minimum global error rate is the foundation for any other improvement for the accuracy rate of the positive class in AdaBoost. The next step is to find the minimum value of $\mathbf{Z}_{t}$ and then get $a_{t}$ under this minimum global error setting.

Theorem 3. Replace the equation in Algorithm 3 with Algorithm 1 to obtain the minimum upper boundary of the global error rate. We have

$$
\alpha_{t}=\frac{1}{2} \ln \left(\frac{\sum_{i: h_{t}\left(\mathbf{x}_{i}\right)=y_{i}} D_{i}^{t} c_{1}^{\left(1-y_{i}\right) / 2}}{\sum_{i: h_{t}\left(\mathbf{x}_{i}\right) \neq y_{i}} D_{i}^{t} c_{1}^{\left(1-y_{i}\right) / 2}}\right)-c_{2} .
$$

For the proof of Theorem 3, see Appendix B.

Remark 3. We know that $\alpha_{t}$ must be positive to make the vote weight parameter reasonable. So, the restriction for $c_{2}$ is

$$
0<c_{2}<\frac{1}{2} \ln \left(\frac{\sum_{i: h_{t}\left(\mathbf{x}_{i}\right)=y_{i} D_{i}^{t} c_{1}^{\left(1-y_{i}\right) / 2}}}{\sum_{i: h_{t}\left(\mathbf{x}_{i}\right) \neq y_{i}} D_{i}^{t} c_{1}^{\left(1-y_{i}\right) / 2}}\right)
$$

The construction of AdaImC algorithmis given in Algorithm 4.

We describe the AdaImC algorithm and show the theoretic proof to support our improvement. The algorithm construction in this section follows the theorems and proofs in previous cost-sensitive AdaBoost algorithms but has no information about determining the cost parameters. The next section tries to build the AdaImC algorithm from a statistical perspective and show the deeper inner connections between the parameters.

\section{A Statistical Approach to AdaImC Algorithm}

Although there is not much theoretic proof for the costsensitive boosting algorithms in the literature, we have convincing statistical studies on the original AdaBoost. This section's basic idea is that the direct proof of AdaImC algorithms is hard to locate so that we show it is identical to the Product of Expert (PoE) model [45] in statistics. All the convincing properties and proofs of the PoE model can support the theorems of the AdaImC algorithm. This section follows the typically statistical approaches $[46,47]$ to the original AdaBoost and shows a statistical perspective to prove our proposed AdaImC algorithm. First, we set the following assumptions.

\section{Assumption 1}

(1) All the observations in the dataset are independent and identically distributed

(2) All the weak classifiers are independent 
Input: the training data: $\left(\mathbf{x}_{1}, y_{1}\right),\left(\mathbf{x}_{2}, y_{2}\right), \ldots,\left(\mathbf{x}_{n}, y_{n}\right)$, where $\mathbf{x}_{i}$ is the feature variable and $y_{i} \in\{-1,1\}$ is the class variable.

(0) Choose the number of weak classifiers $T$. Determine the constants $0<c_{1}<1$, and let $t=1$ to initialize the sample weight $D_{t}(i)=c_{1}^{\left(1-y_{i}\right) / 2}$; determine $0<c_{2}<1 / 2 \ln \left(\sum_{i: h_{t}\left(\mathbf{x}_{i}\right)=y_{i}} D_{i}^{t} c_{1}^{\left(1-y_{i}\right) / 2} / \sum_{i: h_{t}}\left(\mathbf{x}_{i}\right) \neq y_{i} D_{i}^{t} c_{1}^{\left(1-y_{i}\right) / 2}\right), i=1, \ldots, n$;

(1) Under this $D_{t}(i)$, find the weak classifier $h_{t}(\mathbf{x}) \in\{-1,1\}$ minimizing the weighted error rate $\epsilon_{t}=\sum_{h_{t}}\left(\mathbf{x}_{i}\right) \neq y_{i} D_{t}(i)$;

(2) Calculate $\alpha_{t}=(1 / 2) \ln \left(\sum_{i: h_{t}\left(\mathbf{x}_{i}\right)=y_{i}} D_{i}^{t} c_{1}^{\left(1-y_{i}\right) / 2} / \sum_{i: h_{t}\left(\mathbf{x}_{i}\right) \neq y_{i}} D_{i}^{t} c_{1}^{\left(1-y_{i}\right) / 2}\right)-c_{2}$;

(3) Update $D_{t+1}^{i}=\left(\left(c_{1}^{\left(1-y_{i}\right) / 2} D_{t}^{i} \exp \left[-y_{i} h_{t}\left(\mathbf{x}_{i}\right)\left(\alpha_{t}+c_{2}\right)\right]\right) / n \prod_{t=1}^{T} \mathbf{Z}_{t}\right), i=1,2, \ldots, n$, here $\mathbf{Z}_{t}=\sum_{i=1}^{n} D_{t}^{i} c_{1}^{\left(1-y_{i}\right) / 2} \exp \left[-\left(y_{i} h_{t}\left(\mathbf{x}_{i}\right)+c_{2}\right) \alpha_{t}\right]$;

(4) If $t=T$, stop the iteration, else let $t=t+1$ and return to Step 1. Output: the final strong classifier is $H(\mathbf{x})=\operatorname{sign}\left\{\sum_{t=1}^{T} \alpha_{t} h_{t}(\mathbf{x})\right\}$.

Algorithm 4: The AdaImC algorithm.

Every weak classifier is trained based on the data weight related to the previous weak classifiers' performance. So, it is hard to conclude that all the weak classifiers are independent.

Let $\mathbf{X}$ be the random independent variable, and $\mathbf{x}$ is the realization of $\mathbf{X}$. Let $Y$ be the random dependent variable, and $y$ is the realization of $Y$ and $\bar{y}$ is the complement of $y$. We drop the random variables from all the formulas for notational convenience.

Fact 1. From Assumption 1, we have

$$
p\left(y \mid \mathbf{x}, h_{1}, h_{2}, \ldots, h_{J}\right)=\frac{\prod_{j=1}^{T} p\left(y \mid \mathbf{x}, h_{j}\right)}{\prod_{j=1}^{T} p\left(y \mid \mathbf{x}, h_{j}\right)+\prod_{j=1}^{T} p\left(\bar{y} \mid \mathbf{x}, h_{j}\right)},
$$

$$
\begin{aligned}
& p\left(y_{1}, y_{2}, \ldots, y_{n} \mid \mathbf{x}_{1}, \mathbf{x}_{2}, \ldots, \mathbf{x}_{n}, h_{1}(\mathbf{x}), h_{2}(\mathbf{x}), \ldots, h_{j}(\mathbf{x})\right) \\
& \quad=\prod_{i=1}^{n} p\left(y_{i} \mid \mathbf{x}_{i}, h_{1}(\mathbf{x}), h_{2}(\mathbf{x}), \ldots, h_{j}(\mathbf{x})\right)
\end{aligned}
$$

$$
\begin{aligned}
& p\left(y_{1}, y_{2}, \ldots, y_{n} \mid \mathbf{x}_{1}, \mathbf{x}_{2}, \ldots, \mathbf{x}_{n}, h_{1}(\mathbf{x}), h_{2}(\mathbf{x}), \ldots, h_{j-1}(\mathbf{x})\right) \\
& \quad=\prod_{i=1}^{n} p\left(y_{i} \mid \mathbf{x}_{i}, h_{1}(\mathbf{x}), h_{2}(\mathbf{x}), \ldots, h_{j-1}(\mathbf{x})\right) .
\end{aligned}
$$

From Fact 1, we have

$$
p\left(y \mid \mathbf{x}, h_{1}, h_{2}, \ldots, h_{j}\right)=\frac{A}{B+C},
$$

where

$$
\begin{aligned}
& A=p\left(y \mid \mathbf{x}, h_{j}\right) p\left(y \mid \mathbf{x}, h_{1}, h_{2}, \ldots, h_{j-1}\right), \\
& B=p\left(y \mid \mathbf{x}, h_{j}\right) p\left(y \mid \mathbf{x}, h_{1}, h_{2}, \ldots, h_{j-1}\right), \\
& C=p\left(\bar{y} \mid \mathbf{x}, h_{j}\right) p\left(\bar{y} \mid \mathbf{x}, h_{1}, h_{2}, \ldots, h_{j-1}\right) .
\end{aligned}
$$

Our goal is that, with every weak classifier added, we would like the likelihood of the observed data conditioned on the classifiers to increase or remain the same, namely,

$$
\begin{aligned}
& p\left(y_{1}, \ldots, y_{n} \mid \mathbf{x}_{1}, \ldots, \mathbf{x}_{n}, h_{1}(\mathbf{x}), \ldots, h_{j}(\mathbf{x})\right) \\
& \quad \geq p\left(y_{1}, \ldots, y_{n} \mid \mathbf{x}_{1}, \ldots, \mathbf{x}_{n}, h_{1}(\mathbf{x}), \ldots, h_{j-1}(\mathbf{x})\right) .
\end{aligned}
$$

Theorem 4. The sufficient condition of (26) is

$$
\sum_{i=1}^{n} \frac{D_{i}^{j-1}}{p\left(y_{i} \mid \mathbf{x}_{i}, h_{j}\right)} \leq 2
$$

where

$$
D_{i}^{j-1}=\frac{p\left(\overline{y_{i}} \mid \mathbf{x}_{i}, h_{1}, h_{2}, \ldots, h_{j}\right)}{\sum_{i=1}^{n} p\left(\overline{y_{i}} \mid \mathbf{x}_{i}, h_{1}, h_{2}, \ldots, h_{j}\right)} .
$$

For the proof of Theorem 4, see Appendix C.

Using (23), we can simplify (28) as

$$
\begin{aligned}
D_{i}^{j} & =\frac{\left(p\left(\overline{y_{i}} \mid x_{i}, h_{j}\right) p\left(\overline{y_{i}} \mid x_{i}, h_{1}, \ldots, h_{j-1}\right)\right) / Q_{i}^{j}}{\sum_{i=1}^{N}\left(\left(p\left(\overline{y_{i}} \mid x_{i}, h_{j}\right) p\left(\overline{y_{i}} \mid x_{i}, h_{1}, \ldots, h_{j-1}\right)\right) / Q_{i}^{j}\right)} \\
& =\frac{\left(p\left(\overline{y_{i}} \mid x_{i}, h_{j}\right) D_{i}^{j-1}\right) / Q_{i}^{j}}{\sum_{i=1}^{N}\left(p\left(\overline{y_{i}} \mid x_{i}, h_{j}\right) D_{i}^{j-1}\right) / Q_{i}^{j}},
\end{aligned}
$$

where $Q_{i}^{j}$ is the normalization term given by

$$
\begin{aligned}
Q_{i}^{j}= & p\left(y_{i} \mid x_{i}, h_{j}\right) p\left(y_{i} \mid x_{i}, h_{1}, \ldots, h_{j-1}\right) \\
& +p\left(\overline{y_{i}} \mid x_{i}, h_{j}\right) p\left(\overline{y_{i}} \mid x_{i}, h_{1}, \ldots, h_{j-1}\right) .
\end{aligned}
$$

Now, we can build an incremental learning process named the PoE algorithm (Product of Experts model) (Algorithm 5).

The PoE model does not consider the weights of every weak classifier. Also, for considering the cost factor in the model, let us assume the conditional probability contributed by a weak classifier as

$$
\begin{aligned}
p(Y= & y \mid \mathbf{X}=\mathbf{x}, h(\mathbf{x})) \\
= & p(Y=y \mid Z=y) \cdot p(Z=y \mid \mathbf{X}=\mathbf{x}, h(\mathbf{x})) \\
& +p(Y=y \mid Z=\bar{y}) \cdot p(Z=\bar{y} \mid \mathbf{X}=\mathbf{x}, h(\mathbf{x})),
\end{aligned}
$$

where $Z$ is the random variable presenting the prediction made by the classifier. Assume 
Input: the training data: $\left(\mathbf{x}_{1}, y_{1}\right),\left(\mathbf{x}_{2}, y_{2}\right), \ldots,\left(\mathbf{x}_{n}, y_{n}\right)$, where $\mathbf{x}_{i}$ is the feature variable and $y_{i} \in\{-1,1\}$ is the class variable;

(0) Choose the number of weak classifiers $J$, let $j=1$, and initialize the sample weight $D_{i}^{j}=1 / n$;

(1) Under this $D_{i}^{j}$, find the weak classifier $h_{t}(\mathbf{x}) \in\{-1,1\}$ satisfying $\sum_{i=1}^{n}\left(D_{i}^{j} / p\left(y_{i} \mid \mathbf{x}_{i}, h_{j}\right)\right) \leq 2$;

(2) Update $D_{i}^{j+1}=D_{i}^{j} p\left(\bar{y}_{i} \mid \mathbf{x}_{i}, h_{j}\right), D_{i}^{j+1}=D_{i}^{j+1} / \sum_{i}^{n} D_{i}^{j+1}$;

(4) If $j=J$, stop the iteration, else let $j=j+1$ and return to Step 1 .

Output: the final strong classifier is $H(\mathbf{x})=\operatorname{sign}\left\{p\left(y=1 \mid \mathbf{x}, h_{1}, h_{2}, \ldots, h_{J}\right)-p\left(y=-1 \mid \mathbf{x}, h_{1}, h_{2}, \ldots, h_{J}\right)\right\}$.

\section{Algorithm 5: The PoE algorithm.}

$$
\begin{gathered}
p(Y \mid Z=y)=c_{1}^{(1-y) / 2}, \\
p(Y=-1 \mid Z=1)=\frac{p_{e}}{c_{1}}, \\
p(Y=1 \mid Z=-1)=p_{e},
\end{gathered}
$$

where $c_{1}$ is the cost factor, $0<c_{1}<1$, and $p_{e}$ is the classification error probability. This assumption makes the probability of misclassification in the positive class smaller than that in the negative class. From the above assumption, we have

$$
\begin{aligned}
& p(Y=\bar{y} \mid Z=y)=\frac{1+c_{1}}{c_{1}} p_{e}, \\
& p(Y=y \mid Z=y)=c_{1}^{(1-y) / 2}-\frac{1+c_{1}}{c_{1}} p_{e} .
\end{aligned}
$$

Substituting (33) into (31), we have

$$
\begin{aligned}
p(Y= & y \mid \mathbf{X}=\mathbf{x}, h(\mathbf{x})) \\
= & {\left[c_{1}^{(1-y) / 2}-\frac{1+c_{1}}{c_{1}} p_{e}\right] p(Z=y \mid \mathbf{X}=\mathbf{x}, h(\mathbf{x})) } \\
& +\left[\frac{1+c_{1}}{c_{1}} p_{e}\right] p(Z=\bar{y} \mid \mathbf{X}=\mathbf{x}, h(\mathbf{x})) \\
= & {\left[c_{1}^{(1-y) / 2}-\frac{1+c_{1}}{c_{1}} p_{e}\right][1-p(Z=\bar{y} \mid \mathbf{X}=\mathbf{x}, h(\mathbf{x}))] } \\
& +\left[\frac{1+c_{1}}{c_{1}} p_{e}\right] p(Z=\bar{y} \mid \mathbf{X}=\mathbf{x}, h(\mathbf{x})) .
\end{aligned}
$$

Substituting (34) into (26), we have

$$
\sum_{i=1}^{n} \frac{D}{E+F} \leq 2
$$

where

$$
\begin{aligned}
& D=D_{i}^{j-1}, \\
& E=\left[c_{1}^{(1-y) / 2}-\frac{1+c_{1}}{c_{1}} p_{e}^{j}\right]\left[1-p\left(Z=\overline{y_{i}} \mid \mathbf{X}=\mathbf{x}_{i}, h\left(\mathbf{x}_{i}\right)\right)\right], \\
& F=\left[\frac{1+c_{1}}{c_{1}} p_{e}^{j}\right] p\left(Z=\overline{y_{i}} \mid \mathbf{X}=\mathbf{x}_{i}\right) .
\end{aligned}
$$

where $p_{e}^{j}$ is the error parameter of the $j^{\text {th }}$ weak classifier. Simplifying (35) as

$$
\sum_{i: h_{t}\left(\mathbf{x}_{i}\right)=y_{i}} \frac{D_{i}^{j-1}}{c_{1}^{(1-y) / 2}-\left(\left(1+c_{1}\right) / c_{1}\right) p_{e}^{j}}+\sum_{i: h_{t}\left(\mathbf{x}_{i}\right) \neq y_{i}} \frac{D_{i}^{j-1}}{\left(\left(1+c_{1}\right) / c_{1}\right) p_{e}^{j}} \leq 2,
$$

we have

$$
\begin{aligned}
& \sum_{i: h_{t}\left(\mathbf{x}_{i}\right)=y_{i}} \frac{D_{i}^{j-1}}{1-\left(\left(1+c_{1}\right) / c_{1}\right) p_{e}^{j}}+\sum_{i: h_{t}\left(\mathbf{x}_{i}\right) \neq y_{i}} \frac{D_{i}^{j-1}}{\left(\left(1+c_{1}\right) / c_{1}\right) p_{e}^{j}} \\
& \leq \sum_{i: h_{t}\left(\mathbf{x}_{i}\right)=y_{i}} \frac{D_{i}^{j-1}}{c_{1}^{(1-y) / 2}-\left(\left(1+c_{1}\right) / c_{1}\right) p_{e}^{j}} \\
& +\sum_{i: h_{t}\left(\mathbf{x}_{i}\right) \neq y_{i}} \frac{D_{i}^{j-1}}{\left(\left(1+c_{1}\right) / c_{1}\right) p_{e}^{j}} \leq 2 .
\end{aligned}
$$

Set $\epsilon_{j}=\sum_{i: h_{t}\left(\mathbf{x}_{i}\right) \neq y_{i}} D_{i}^{j-1}$ and $\epsilon_{j}^{c}=\sum_{i: h_{t}\left(\mathbf{x}_{i}\right) \neq y_{i}} c_{1}^{\left(1-y_{i}\right) / 2} D_{i}^{j-1}$, and then we have

$$
\epsilon_{j}^{c} \leq \epsilon_{j} \leq \frac{1+c_{1}}{c_{1}} p_{e}^{j} \leq \frac{1}{2}
$$

Parameter $p_{e}^{j}$ is defined as

$$
p_{e}^{j}=\frac{c_{1} \sum_{i} c_{1}^{\left(1-y_{i}\right) / 2}}{1+c_{1}}\left[\frac{e^{-\left(\alpha_{j}+c_{2}\right)}}{e^{\alpha_{j}+c_{2}}+e^{-\left(\alpha_{j}+c_{2}\right)}}\right]
$$

where $c_{2}$ is positive.

Lemma 2. When $c_{1} \leq\left(p_{e}^{j} /\left(\epsilon_{j}^{c}-p_{e}^{j}\right)\right)$ and $c_{2} \leq(1 / 2) \ln$ $\left(\left(\sum_{i} c_{1}^{\left(1-y_{i}\right) / 2} / \epsilon_{j}^{c}\right)-1\right)$, choose the lower boundary $P_{e}^{j}=\left(c_{1} /\left(1+c_{1}\right)\right) \epsilon_{j}^{c}$ to obtain $\alpha_{j}$ from (40):

$$
\begin{aligned}
\alpha_{j} & =\frac{1}{2} \ln \left[\frac{\sum_{i} c_{1}^{\left(1-y_{i}\right) / 2}-\epsilon_{j}^{c}}{\epsilon_{j}^{c}}\right]-c_{2} \\
& =\frac{1}{2} \ln \left[\frac{\left.\sum_{i: h_{t}\left(\mathbf{x}_{i}\right)=y_{i} D_{i}^{t} c_{1}^{\left(1-y_{i}\right) / 2}} \sum_{i: h_{t}\left(\mathbf{x}_{i}\right) \neq y_{i} D_{i}^{t} c_{1}^{\left(1-y_{i}\right) / 2}}\right]-c_{2} .}{} .\right.
\end{aligned}
$$

In Lemma $2, c_{1} \leq\left(p_{e}^{j} /\left(\epsilon_{j}^{c}-p_{e}^{j}\right)\right)$ can ensure that the choice of $p_{e}^{j}$ satisfies $(40)$ and $c_{2} \leq(1 / 2) \ln \left(\left(\sum_{i} c_{1}^{\left(1-y_{i}\right) / 2} / \epsilon_{j}^{c}\right)-\right.$ $1)$ is necessary for the positive property of $\alpha_{j}$. 
The value of $p(Y=\bar{y} \mid \mathbf{X}=\mathbf{x}, h(\mathbf{x}))$ can be computed based on (33):

$$
\begin{aligned}
p & (Y=\bar{y} \mid \mathbf{X}=\mathbf{x}, h(\mathbf{x})) \\
= & {\left[c_{1}^{(1-y) / 2}-\frac{1+c_{1}}{c_{1}} p_{e}^{j}\right] p(Z=\bar{y} \mid \mathbf{X}=\mathbf{x}, h(\mathbf{x})) } \\
& +\left[\frac{1+c_{1}}{c_{1}} p_{e}^{j}\right]\left[c_{1}^{(1-y) / 2}-p(Z=\bar{y} \mid \mathbf{X}=\mathbf{x}, h(\mathbf{x}))\right] \\
= & \begin{cases}\frac{1+c_{1}}{c_{1}} p_{e}^{j}, & \text { if } h(\mathbf{x})=y, \\
c_{1}^{(1-y) / 2}-\frac{1+c_{1}}{c_{1}} p_{e}^{j}, & \text { if } h(\mathbf{x}) \neq y .\end{cases}
\end{aligned}
$$

Substituting the value of $p_{e}^{j}$ given in (40) into (42), we have

$$
\begin{aligned}
& p\left(\overline{y_{i}} \mid \mathbf{x}_{i}, h\left(\mathbf{x}_{i}\right)\right) \\
& = \begin{cases}c_{1}^{\left(1-y_{i}\right) / 2}\left[\frac{e^{-\left(\alpha_{j}+c_{2}\right)}}{\left.e^{\alpha_{j}+c_{2}}+e^{-\left(\alpha_{j}+c_{2}\right)}\right]}\right. & \text { if } h(\mathbf{x})=y, \\
c_{1}^{\left(1-y_{i}\right) / 2}-c_{1}^{\left(1-y_{i}\right) / 2}\left[\frac{e^{-\left(\alpha_{j}+c_{2}\right)}}{\left.e^{\alpha_{j}+c_{2}}+e^{-\left(\alpha_{j}+c_{2}\right)}\right]}\right. & \text { if } h(\mathbf{x}) \neq y,\end{cases} \\
& =\frac{c_{1}^{\left(1-y_{i}\right) / 2} e^{\left[-y_{i} h_{j}\left(\mathbf{x}_{i}\right)\left(\alpha_{j}+c_{2}\right)\right]}}{e^{\alpha_{j}+c_{2}}+e^{-\left(\alpha_{j}+c_{2}\right)}} .
\end{aligned}
$$

Recalling (29), we have

$$
D_{i}^{j}=\frac{\left(c_{1}^{\left(1-y_{i}\right) / 2} e^{\left[-y_{i} h_{j}\left(\mathbf{x}_{i}\right)\left(\alpha_{j}+c_{2}\right)\right]} D_{i}^{j-1}\right) / Q_{i}^{j}}{\sum_{i=1}^{N}\left(c_{1}^{\left(1-y_{i}\right) / 2} e^{\left[-y_{i} h_{j}\left(\mathbf{x}_{i}\right)\left(\alpha_{j}+c_{2}\right)\right]} D_{i}^{j-1}\right) / Q_{i}^{j}},
$$

where $Q_{i}^{j}$ is the normalization term given by

$$
\begin{aligned}
Q_{i}^{j}= & c_{1}^{\left(1-y_{i}\right) / 2} e^{\left[-y_{i} h_{j}\left(\mathbf{x}_{i}\right)\left(\alpha_{j}+c_{2}\right)\right]} p\left(\overline{y_{i}} \mid \mathbf{x}_{i}, h_{1}\left(\mathbf{x}_{i}\right), \ldots, h_{j-1}\left(\mathbf{x}_{i}\right)\right) \\
& +c_{1}^{\left(1-y_{i}\right) / 2} e^{\left[y_{i} h_{j}\left(\mathbf{x}_{i}\right)\left(\alpha_{j}+c_{2}\right)\right]} p\left(y_{i} \mid \mathbf{x}_{i}, h_{1}\left(\mathbf{x}_{i}\right), \ldots, h_{j-1}\left(\mathbf{x}_{i}\right)\right) .
\end{aligned}
$$

Now, we have given a statistical perspective to see our proposed Algorithm 4. However, the statistical approach gives us some deeper information about this algorithm. Lemma 2 gives the conditions of $c_{1} \leq\left(p_{e}^{j} /\left(\epsilon_{j}^{c}-p_{e}^{j}\right)\right)$ and $c_{2} \leq(1 / 2) \ln \left(\left(\sum_{i} c_{1}^{\left(1-y_{i}\right) / 2} / \epsilon_{j}^{c}\right)-1\right)$. The choice of $c_{1}$ and $c_{2}$ hasto satisfy these assumptions in our presented algorithm.

\section{Numerical Studies}

5.1. Simulation Studies. This section illustrates how to determine the cost parameters $c_{1}$ and $c_{2}$ in our model. A simulated dataset is employed to test our proposed model's performance.

5.1.1. Datasets. Five two-dimensional simulated Gaussian data are chosen, and they are distributed as

$$
\mathbf{X}_{i j}=\left(\begin{array}{c}
X_{i j 1} \\
X_{i j 2}
\end{array}\right) \stackrel{\text { indep }}{\sim} \mathcal{N}_{2}\left(\boldsymbol{\mu}_{i}, \boldsymbol{\Sigma}_{i}\right),
$$

where $i=-1,1$ and $j=1, \ldots, n_{i}$. We set

$$
\begin{aligned}
\mathbf{u}_{-1} & =\left(\begin{array}{l}
7 \\
8
\end{array}\right), \\
\mathbf{u}_{1} & =\left(\begin{array}{l}
13 \\
15
\end{array}\right), \\
\boldsymbol{\Sigma}_{-1} & =\left(\begin{array}{ll}
10 & 3 \\
3 & 8
\end{array}\right), \\
\boldsymbol{\Sigma}_{1} & =\left(\begin{array}{ll}
1 & 0 \\
0 & 2
\end{array}\right) .
\end{aligned}
$$

All the variables in $\mathbf{X}_{-1, j}$ and $\mathbf{X}_{1, j}$ have the class labels -1 (majority) and 1 (minority), respectively. We set five kinds of sizes, $\quad\left(n_{p}, n_{n}\right)=(100,100),(50,100),(25,100),(10,100)$, and $(5,100)$, to illustrate the performance of different algorithms in different-sized data. $b=1,2,4,10,20$ for these five cases. A larger $b$ indicates a more severely imbalanced dataset. Figure 2 shows the training datasets.

5.1.2. Parameters Setup. The statistical analysis shows that the cost parameters $c_{1}$ and $c_{2}$ have to satisfy

$$
\begin{aligned}
c_{1} & \leq \frac{p_{e}^{j}}{(1 / 2)-p_{e}^{j}} \leq \frac{p_{e}^{j}}{\epsilon_{j}^{c}-p_{e}^{j},} \\
c_{2} & \leq \frac{1}{2} \ln \left(2 n c_{1}-1\right) \\
& \leq \frac{1}{2} \ln \left(2 \sum_{i} c_{1}^{\left(1-y_{i}\right) / 2}-1\right) \leq \frac{1}{2} \ln \left(\frac{\sum_{i} c_{1}^{\left(1-y_{i}\right) / 2}}{\epsilon_{j}^{c}}-1\right) .
\end{aligned}
$$

We set the upper boundaries as the chosen values of $c_{1}$ and $c_{2}$ in (48) and (49). The restrictions of $\epsilon_{j}^{c}<(1 / 2)$ and $c_{1} \leq 1$ indicate $p_{e}^{j} \leq(1 / 2) \epsilon_{j}^{c} \leq(1 / 4)$. Equation (49) requires that $2 n c_{1}-1>0$, which indicates $c_{1}>(1 / 2 n)$.

$\mathrm{We}$ choose the values of $p_{e}^{j}$ as $(1 / 4),(1 / 8),(1 / 16),(1 / 32),(1 / 64)$, and (1/128) to ensure that both cost parameters have the values. Table 4 lists the cost parameters in the present five simulated datasets.

5.1.3. Evaluation Criteria. Twelve criteria listed in Table 5 are used to evaluate the performance of algorithms in this paper, including the error rate criteria $r_{e}$ and the misclassified cost criteria $c_{e}$.

The calculations of $r_{e_{e}}^{\text {test }}, r_{e_{p}}^{\text {test }}$, and $c_{e}^{\text {test }}$ use the same-sized test data as the training data, $n^{\text {train }}=n^{\text {test }}=n, n_{p}^{\text {train }}=$ 


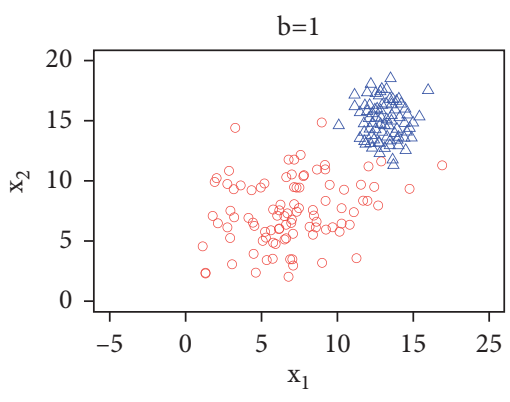

$\triangle$ Nagetive Class $n_{n}=100$
$\triangle$ Positive Class $n_{p}=100$

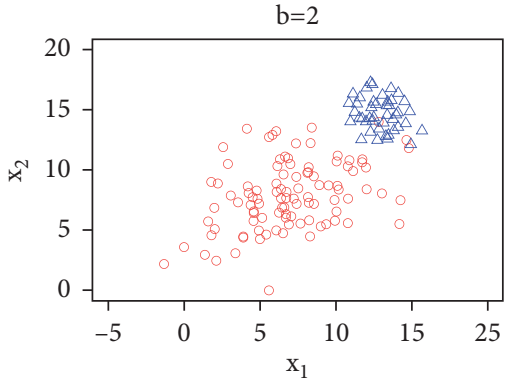

○ Nagetive Class $n_{n}=100$

$\triangle$ Positive Class $n_{p}=50$

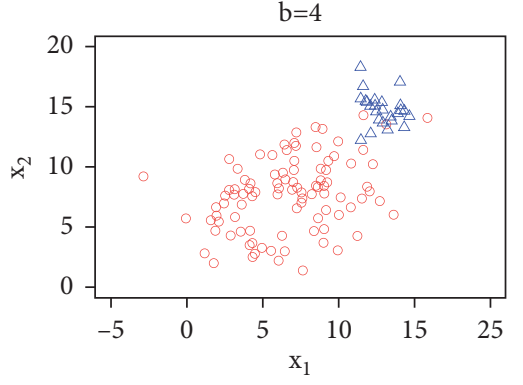

- Nagetive Class $n_{n}=100$

$\triangle$ Positive Class $n_{p}=25$

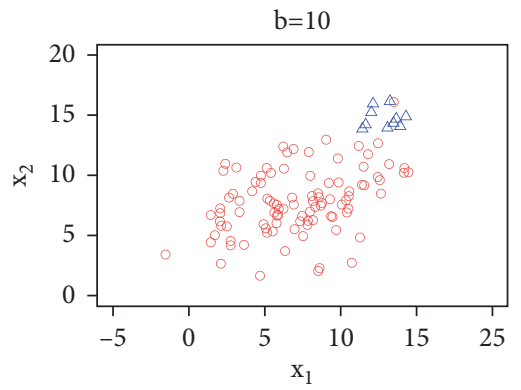

- Nagetive Class $n_{n}=100$

$\triangle$ Positive Class $n_{p}=10$

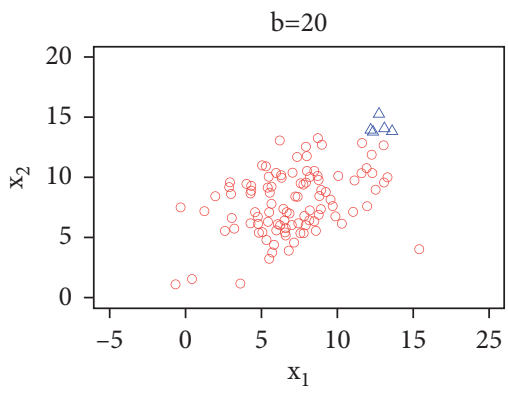

- Nagetive Class $n_{n}=100$

$\triangle$ Positive Class $n_{p}=50$

FIgURE 2: Simulated Gaussian data for numeric studies.

$n_{p}^{\text {test }}=n_{p}$. Small-sized and large-sized test data are used for the calculations of $r_{e_{g}}^{\text {stest }}, r_{e_{p}}^{\text {stest }}$, and $c_{e_{g}}^{\text {stest }}$ and $r_{e_{g_{g}}}^{\text {ltest }}, r_{e_{p}}^{\text {ltest }}$, and $c_{e_{g}}^{\text {ltest }}$, respectively. It means $n^{s}<n<n^{l}, n_{p}^{s}<n_{p}<n_{p}$. Note that the positive class error cost is equal to the error rate since the positive cost parameter is set as 1 . All the test datasets in this paper keep the same imbalance index $b$ as the training data, namely,

$$
\frac{\left|\mathbf{S}_{-}^{\text {test }}\right|}{\left|\mathbf{S}_{+}^{\text {test }}\right|}=\frac{\left|\mathbf{S}_{-}^{\text {train }}\right|}{\left|\mathbf{S}_{+}^{\text {train }}\right|}=b .
$$

5.1.4. Results of Simulation Studies. We run every simulated dataset 100 times and calculate the mean and standard deviation values as the results. Tables $6-10$ list the experiments. Obviously, with the imbalanced index $b$ growing larger, the smaller cost parameters could better classify performances. We conjecture that the classification performance is best when the corresponding $c_{1}$ is closest to $1 / b$.

5.2. Real Data Studies. Four real binary datasets are studied, and the values of their imbalanced index $b$ are 15.13, 11.59, 5.14, and 3.36. Since all the datasets are imbalanced, each dataset is randomly split in half as the training part and half as the test part to avoid the situation that there are no or too few positive samples in the training or test data. We choose thirteen popular classification algorithms in Table 11 for comparisons. The first five are all the traditional ones and the sixth, PRVM [48], is designed for the imbalanced data problem, which has achieved convincing results. The last seven ones are the traditional cost-sensitive classifiers, which we have discussed in the discussion part. Note that the AdaImC algorithm in this part is tested based on the different determinations of the cost factors to choose the optimal parameter setup before joining the comparison. We will not show this process again in the real data studies.

5.2.1. Seismic-Bumps Data Study. The seismic-bumps data with $b=15.13$ are obtained from the UCI Machine Learning Repository [49]. This dataset is about the seismic hazard in mining activity. Mining activity is always connected with the occurrence of dangers, which are called mining hazards. Seismic hazard is a special and dangerous case in mining hazards. The seismic-bumps dataset is intensely imbalanced with only 170 samples of the positive class among a total of 2584 samples, and it contains 18 explanatory variables, including seismic hazard assessment obtained by different methods, seismic energy recorded by different types of equipment, and the number of seismic bumps in different energy range. The hazardous state is the negative class defined as a high energy seismic bump occurring in the next shift, which is indicated by $y=-1$. The nonhazardous state is the positive class defined as no high energy seismic bump occurring in the next shift, which is indicated by $y=1$.

Table 12 displays the seismic-bumps data experiment. It shows the great performance of our proposed algorithms for the classification of this dataset. Except for the specificity, all evaluation criteria of the PRVM and the AdaImC perform 
TABLe 4: The cost parameter determinations.

\begin{tabular}{|c|c|c|c|c|c|c|c|}
\hline & $p_{e}^{j}$ & $1 / 4$ & $1 / 8$ & $1 / 16$ & $1 / 32$ & $1 / 64$ & $1 / 128$ \\
\hline & $c_{1}$ & 1 & $1 / 3$ & $1 / 7$ & $1 / 15$ & $1 / 31$ & $1 / 63$ \\
\hline & $b=1$ & 2.9945 & 2.4427 & 2.0140 & 1.6226 & 1.2384 & 0.8385 \\
\hline & $b=2$ & 2.8502 & 2.2976 & 1.8671 & 1.4722 & 1.0804 & 0.6625 \\
\hline$c_{2}$ & $b=4$ & 2.7587 & 2.2054 & 1.7736 & 1.3758 & 0.9775 & 0.5440 \\
\hline & $b=10$ & 2.6945 & 2.1406 & 1.7077 & 1.3075 & 0.9039 & 0.4566 \\
\hline & $b=20$ & 2.6712 & 2.1171 & 1.6836 & 1.2825 & 0.8767 & 0.4236 \\
\hline
\end{tabular}

TABle 5: The criteria for classification evaluation.

Training Data Global Error Rate

Training data positive class error rate

Training data global error cost

Same size test data global error rate

Same size test data positive class error rate

Same size test data global error cost

Smaller size test data global error rate

Smaller size test data positive class error rate

Smaller size test data global error cost

Larger size test data global error rate

Larger size test data positive class error rate

Larger size test data global error cost

$r_{e_{g}}^{\text {train }}=\left(\left|y_{i}^{\text {train }} \neq y_{i_{*}}^{\text {train }}\right| / n\right)$
$r_{e_{p}}^{\text {train }}=\left(\left|y_{i}^{\text {train }} \neq y_{i_{*}}^{\text {train }} \& y_{i}^{\text {train }}=1\right| / n_{p}\right)$
$c_{e_{g}}^{\text {train }}=\left(c_{i} \cdot\left|y_{i}^{\text {train }} \neq y_{i_{*}}^{\text {train }}\right| / \sum_{i} c_{i}\right)$
$r_{e_{g}}^{\text {test }}=\left(\left|y_{i}^{\text {test }} \neq y_{i_{*}}^{\text {test }}\right| / n\right)$
$r_{e_{p}}^{\text {test }}=\left(\left|y_{i}^{\text {test }} \neq y_{i_{*}}^{\text {test }} \& y_{i}^{\text {test }}=1\right| / n_{p}\right)$
$c_{e_{g}}^{\text {test }}=\left(c_{i} \cdot\left|y_{i}^{\text {test }} \neq y_{i *}^{\text {test }}\right|\right) / \sum_{i} c_{i}$
$r_{e_{g}}^{\text {stest }}=\left(\left|y_{i}^{\text {stest }} \neq y_{i_{*}}^{\text {stest }}\right| / n^{s}\right)$
$r_{e_{p}}^{\text {stest }}=\left(\left|y_{i}^{\text {stest }} \neq y_{i_{*}}^{\text {stest }} \& y_{i}^{\text {stest }}=1\right| / n_{p}^{s}\right)$
$c_{e_{g}}^{\text {stest }}=\left(\left(c_{i} \cdot\left|y_{i}^{\text {stest }} \neq y_{i_{*}}^{\text {stest }}\right|\right) / \sum_{i} c_{i}\right)$
$r_{e_{g}}^{\text {test }}=\left(\left|y_{i}^{\text {ltest }} \neq y_{i_{*}}^{\text {lest }}\right| / n^{l}\right)$
$r_{e_{p}}^{\text {ltest }}=\left(\left|y_{i}^{\text {ltest }} \neq y_{i *}^{\text {ltest }} \& y_{i}^{\text {ltest }}=1\right| / n_{p}^{l}\right)$
$c_{e_{g}}^{\text {ltest }}=\left(\left(c_{i} \cdot\left|y_{i}^{\text {ltest }} \neq y_{i_{*}}^{\text {ltest }}\right|\right) / \sum_{i} c_{i}\right)$

TABLE 6: The experiment results of $b=1$.

\begin{tabular}{|c|c|c|c|c|c|c|}
\hline & $c_{1}=1 c_{2}=2.9945$ & $c_{1}=1 / 3 c_{2}=2.4427$ & $c_{1}=1 / 7 c_{2}=2.0140$ & $c_{1}=1 / 15 c_{2}=1.6226$ & $c_{1}=1 / 31 c_{2}=1.2384$ & $c_{1}=1 / 63 c_{2}=0.8385$ \\
\hline$r_{e}^{\text {train }}$ & $0.0030(0.0438)$ & $0.0036(0.0393)$ & $0.0035(0.0310)$ & $0.0039(0.0334)$ & $0.0040(0.0554)$ & $0.0042(0.0612)$ \\
\hline$r_{e_{p}}^{g}$ & $0.0031(0.0448)$ & $0.0034(0.0329)$ & $0.0036(0.0426)$ & $0.0039(0.0381)$ & $0.0040(0.0796)$ & $0.0041(0.0540)$ \\
\hline$c_{e_{g}}^{\text {train }}$ & $0.0030(0.0318)$ & $0.0034(0.0538)$ & $0.0032(0.0685)$ & $0.0035(0.0317)$ & $0.0036(0.0696)$ & $0.0038(0.0745)$ \\
\hline$r_{e_{g}}^{g}$ & $0.0031(0.0339)$ & $0.0031(0.0376)$ & $0.0032(0.0657)$ & $0.0037(0.0331)$ & $0.0039(0.0300)$ & $0.0040(0.0496)$ \\
\hline$r_{e_{p}}^{g}$ & $0.0031(0.0323)$ & $0.0036(0.0537)$ & $0.0030(0.0849)$ & $0.0038(0.0415)$ & $0.0039(0.0338)$ & $0.0041(0.0900)$ \\
\hline$c_{e_{g}}^{c_{p}}$ & $0.0031(0.0408)$ & $0.0030(0.0644)$ & $0.0031(0.0593)$ & $0.0035(0.0595)$ & $0.0038(0.0384)$ & $0.0039(0.0563)$ \\
\hline$r_{e_{a}}^{\text {stest }}$ & $0.0031(0.0477)$ & $0.0033(0.0333)$ & $0.0032(0.0640)$ & $0.0037(0.0446)$ & $0.0039(0.0755)$ & $0.0042(0.0620)$ \\
\hline$r_{e_{p}}^{s}$ & $0.0031(0.0332)$ & $0.0033(0.0346)$ & $0.0030(0.0748)$ & $0.0039(0.0536)$ & $0.0041(0.0709)$ & $0.0042(0.0657)$ \\
\hline$c_{e_{g}}^{p p}$ & $0.0030(0.0439)$ & $0.0032(0.0572)$ & $0.0031(0.0554)$ & $0.0035(0.0390)$ & $0.0036(0.0420)$ & $0.0040(0.0565)$ \\
\hline$r_{e_{q}}^{g}$ & $0.0029(0.0323)$ & $0.0035(0.0780)$ & $0.0031(0.0437)$ & $0.0039(0.0667)$ & $0.0040(0.0434)$ & $0.00041(0.0887)$ \\
\hline$r_{e_{p}}^{g}$ & $0.0030(0.0478)$ & $0.0031(0.0365)$ & $0.0032(0.0776)$ & $0.0038(0.0662)$ & $0.0040(0.0835)$ & $0.0043(0.0499)$ \\
\hline$c_{e_{g}}^{\text {ltest }}$ & $0.0029(0.0351)$ & $0.0034(0.0522)$ & $0.0030(0.0676)$ & $0.0037(0.0334)$ & $0.0038(0.0341)$ & $0.0040(0.0344)$ \\
\hline
\end{tabular}

TABLe 7: The experiment results of $b=2$.

\begin{tabular}{|c|c|c|c|c|c|c|}
\hline & $c_{1}=1 c_{2}=2.9945$ & $c_{1}=1 / 3 c_{2}=2.4427$ & $c_{1}=1 / 7 c_{2}=2.0140$ & $c_{1}=1 / 15 c_{2}=1.6226$ & $c_{1}=1 / 31 c_{2}=1.2384$ & $c_{1}=1 / 63 c_{2}=0.8385$ \\
\hline$r_{e_{q}}^{\text {train }}$ & $0.0043(0.4673)$ & $0.0020(0.4204)$ & $0.0027(0.4370)$ & $0.0026(0.4246)$ & $0.0029(0.3300)$ & $0.0033(0.3555)$ \\
\hline$r_{e_{p}}^{\text {train }}$ & $0.0053(0.3950)$ & $0.0026(0.4368)$ & $0.0025(0.4637)$ & $0.0026(0.4645)$ & $0.0026(0.3891)$ & $0.0029(0.4967)$ \\
\hline$c_{e_{g}}^{\text {train }}$ & $0.0043(0.4082)$ & $0.0022(0.3104)$ & $0.0024(0.4686)$ & $0.0024(0.4837)$ & $0.0027(0.4853)$ & $0.0030(0.3063)$ \\
\hline$r_{e_{g}}^{\text {test }}$ & $0.0038(0.3738)$ & $0.0021(0.4547)$ & $0.0035(0.4430)$ & $0.0027(0.3696)$ & $0.0029(0.3119)$ & $0.0029(0.4192)$ \\
\hline$r_{e_{p}}^{\text {test }}$ & $0.0045(0.3145)$ & $0.0028(0.4728)$ & $0.0025(0.4966)$ & $0.0028(0.3499)$ & $0.0031(0.4755)$ & $0.0033(0.4287)$ \\
\hline$c_{e_{g}}^{\text {test }}$ & $0.0038(0.4071)$ & $0.0020(0.4748)$ & $0.0029(0.4787)$ & $0.0027(0.3140)$ & $0.0029(0.4898)$ & $0.0029(0.4157)$ \\
\hline$r_{e_{g}}^{\text {stest }}$ & $0.0042(0.3660)$ & $0.0016(0.3660)$ & $0.0024(0.3477)$ & $0.0029(0.4636)$ & $0.0029(0.4353)$ & $0.0031(0.3849)$ \\
\hline$r_{e_{p}}^{\text {stest }}$ & $0.0055(0.4836)$ & $0.0018(0.3095)$ & $0.0032(0.3323)$ & $0.0032(0.3091)$ & $0.0026(0.4430)$ & $0.0031(0.4331)$ \\
\hline$c_{e_{g}}^{\text {stest }}$ & $0.0042(0.3592)$ & $0.0025(0.4214)$ & $0.0022(0.4206)$ & $0.0026(0.4411)$ & $0.0028(0.3444)$ & $0.0028(0.3741)$ \\
\hline$r_{e_{g}}^{\text {ltest }}$ & $0.0042(0.3248)$ & $0.0018(0.3845)$ & $0.0026(0.4646)$ & $0.0024(0.4188)$ & $0.0028(0.4434)$ & $0.0033(0.3432)$ \\
\hline$r_{e_{p}}^{\text {ltest }}$ & $0.0054(0.4604)$ & $0.0020(0.3554)$ & $0.0026(0.3905)$ & $0.0025(0.3730)$ & $0.0031(0.3185)$ & $0.0030(0.3504)$ \\
\hline$c_{e_{g}}^{1 \text { lest }}$ & $0.0042(0.4394)$ & $0.0018(0.3538)$ & $0.0025(0.3312)$ & $0.0023(0.3973)$ & $0.0027(0.3230)$ & $0.0031(0.4799)$ \\
\hline
\end{tabular}


TABLE 8: The experiment results of $b=5$.

\begin{tabular}{|c|c|c|c|c|c|c|}
\hline & $c_{1}=1 c_{2}=2.9945$ & $c_{1}=1 / 3 c_{2}=2.4427$ & $c_{1}=1 / 7 c_{2}=2.0140$ & $c_{1}=1 / 15 c_{2}=1.6226$ & $c_{1}=1 / 31 c_{2}=1.2384$ & $c_{1}=1 / 63 c_{2}=0.8385$ \\
\hline$r_{e_{g}}^{\text {train }}$ & $0.0062(0.4142)$ & $0.0032(0.3957)$ & $0.0025(0.3406)$ & $0.0031(0.2384)$ & $0.0032(0.2024)$ & $0.0032(0.2019)$ \\
\hline$r_{e_{p}}^{\text {train }}$ & $0.0089(0.3637)$ & $0.0050(0.4967)$ & $0.0030(0.4904)$ & $0.0031(0.3888)$ & $0.0031(0.4530)$ & $0.0033(0.2667)$ \\
\hline$c_{e_{g}}^{\text {train }}$ & $0.0062(0.2088)$ & $0.0030(0.2824)$ & $0.0024(0.4008)$ & $0.0030(0.3674)$ & $0.0031(0.2040)$ & $0.0031(0.3920)$ \\
\hline$r_{e_{g}}^{\text {test }}$ & $0.0061(0.2904)$ & $0.0028(0.2729)$ & $0.0026(0.4036)$ & $0.0030(0.4861)$ & $0.0030(0.4195)$ & $0.0033(0.4460)$ \\
\hline$r_{e_{p}}^{\text {test }}$ & $0.0090(0.4453)$ & $0.0058(0.3510)$ & $0.0027(0.3723)$ & $0.0033(0.3545)$ & $0.0032(0.2935)$ & $0.0033(0.2351)$ \\
\hline$c_{e_{g}}^{p e s t}$ & $0.0061(0.3162)$ & $0.0027(0.2519)$ & $0.0024(0.4383)$ & $0.0029(0.3922)$ & $0.0028(0.3349)$ & $0.0031(0.2821)$ \\
\hline$r_{e_{g}}^{g}$ & $0.0063(0.4622)$ & $0.0028(0.4731)$ & $0.0029(0.4915)$ & $0.0033(0.4227)$ & $0.0031(0.3523)$ & $0.0031(0.3897)$ \\
\hline$r_{e_{p}}^{\text {stest }}$ & $0.0093(0.4590)$ & $0.0051(0.4006)$ & $0.0025(0.3267)$ & $0.0033(0.3091)$ & $0.0032(0.3934)$ & $0.0033(0.2366)$ \\
\hline$c_{e_{g}}^{\text {stest }}$ & $0.0063(0.4888)$ & $0.0027(0.3809)$ & $0.0027(0.3463)$ & $0.0030(0.2130)$ & $0.0030(0.4935)$ & $0.0028(0.4646)$ \\
\hline$r_{e_{q}}^{\text {ltest }}$ & $0.0068(0.3685)$ & $0.0032(0.2169)$ & $0.0026(0.2913)$ & $0.0030(0.4536)$ & $0.0032(0.4427)$ & $0.0031(0.3334)$ \\
\hline$r_{e_{p}}^{l \text { lest }}$ & $0.0088(0.4268)$ & $0.0050(0.2679)$ & $0.0030(0.3359)$ & $0.0033(0.2583)$ & $0.0031(0.3136)$ & $0.0031(0.2392)$ \\
\hline$c_{e_{g}}^{\text {ltest }}$ & $0.0068(0.2535)$ & $0.0029(0.3750)$ & $0.0024(0.2735)$ & $0.0028(0.3600)$ & $0.0030(0.3842)$ & $0.0030(0.4166)$ \\
\hline
\end{tabular}

Table 9: The experiment results of $b=10$.

\begin{tabular}{|c|c|c|c|c|c|c|}
\hline & $c_{1}=1 c_{2}=2.9945$ & $c_{1}=1 / 3 c_{2}=2.4427$ & $c_{1}=1 / 7 c_{2}=2.0140$ & $c_{1}=1 / 15 c_{2}=1.6226$ & $c_{1}=1 / 31 c_{2}=1.2384$ & $c_{1}=1 / 63 c_{2}=0.8385$ \\
\hline$r_{e_{e}}^{\text {train }}$ & $0.2131(0.3640)$ & $0.0629(0.3041)$ & $0.0029(0.5293)$ & $0.0028(0.3091)$ & $0.0029(0.3091)$ & $0.0029(0.3396)$ \\
\hline$r_{e_{p}}^{\operatorname{train}^{\prime}}$ & $0.4229(0.5814)$ & $0.2629(0.4365)$ & $0.0029(0.4660)$ & $0.0028(0.5930)$ & $0.0028(0.5930)$ & $0.0031(0.5406)$ \\
\hline$c_{e_{g}}^{\text {train }}$ & $0.2030(0.4871)$ & $0.0630(0.5596)$ & $0.0028(0.5697)$ & $0.0030(0.3245)$ & $0.0030(0.3245)$ & $0.0030(0.5110)$ \\
\hline$r_{e_{g}}^{g}$ & $0.2533(0.2023)$ & $0.0630(0.4073)$ & $0.0030(0.3995)$ & $0.0028(0.2032)$ & $0.0028(0.2032)$ & $0.0027(0.4745)$ \\
\hline$r_{e_{p}}^{\text {test }}$ & $0.4531(0.5172)$ & $0.2630(0.3888)$ & $0.0028(0.3106)$ & $0.0030(0.4138)$ & $0.0030(0.4138)$ & $0.0028(0.4054)$ \\
\hline$c_{e_{g}}^{\text {test }}$ & $0.2629(0.5811)$ & $0.0631(0.4503)$ & $0.0029(0.5217)$ & $0.0028(0.4158)$ & $0.0028(0.4158)$ & $0.0033(0.2670)$ \\
\hline$r_{e_{g}}^{g}$ & $0.2530(0.4356)$ & $0.0629(0.4585)$ & $0.0030(0.3506)$ & $0.0030(0.2349)$ & $0.0030(0.2349)$ & $0.0033(0.4934)$ \\
\hline$r_{e_{p}}^{e_{g}}$ & $0.4230(0.3356)$ & $0.2629(0.2448)$ & $0.0029(0.5382)$ & $0.0029(0.3647)$ & $0.0030(0.3647)$ & $0.0033(0.5974)$ \\
\hline$c_{e_{g}}^{\text {stest }}$ & $0.2231(0.3216)$ & $0.0630(0.3403)$ & $0.0030(0.5239)$ & $0.0028(0.3297)$ & $0.0028(0.3297)$ & $0.0033(0.4633)$ \\
\hline$r_{e_{a}}^{g}$ & $0.3029(0.3406)$ & $0.0629(0.4907)$ & $0.0028(0.3624)$ & $0.0029(0.5111)$ & $0.0029(0.5111)$ & $0.0030(0.3396)$ \\
\hline$r_{e_{p}}^{g}$ & $0.4031(0.2818)$ & $0.2629(0.2790)$ & $0.0029(0.2387)$ & $0.0028(0.5565)$ & $0.0029(0.5565)$ & $0.0030(0.3725)$ \\
\hline$c_{e_{g}}^{\text {ltest }}$ & $0.3030(0.5284)$ & $0.0629(0.5398)$ & $0.0029(0.3888)$ & $0.0027(0.4037)$ & $0.0027(0.4037)$ & $0.0028(0.5488)$ \\
\hline
\end{tabular}

Table 10: The experiment results of $b=20$.

\begin{tabular}{lcccccc}
\hline & $c_{1}=1 c_{2}=2.9945$ & $c_{1}=1 / 3 c_{2}=2.4427$ & $c_{1}=1 / 7 c_{2}=2.0140$ & $c_{1}=1 / 15 c_{2}=1.6226$ & $c_{1}=1 / 31 c_{2}=1.2384$ & $c_{1}=1 / 63 c_{2}=0.8385$ \\
\hline$r_{e_{g}}^{\text {train }}$ & $0.2032(0.2626)$ & $0.1233(0.3151)$ & $0.0829(0.4914)$ & $0.0030(0.5119)$ & $\mathbf{0 . 0 0 2 9}(\mathbf{0 . 4 9 1 1})$ & $0.0033(0.5357)$ \\
$r_{e_{p}}^{\text {train }}$ & $0.5730(0.2680)$ & $0.3132(0.4644)$ & $0.1330(0.3304)$ & $\mathbf{0 . 0 0 2 6 ( \mathbf { 0 . 5 6 5 5 } )}$ & $\mathbf{0 . 0 0 2 6}(\mathbf{0 . 2 4 0 2})$ & $0.0029(0.3079)$ \\
$c_{e_{g}}^{\text {train }}$ & $0.2033(0.2633)$ & $0.1232(0.4608)$ & $0.0833(0.4054)$ & $0.0030(0.2351)$ & $\mathbf{0 . 0 0 2 7}(\mathbf{0 . 2 1 4 7})$ & $0.0030(0.5034)$ \\
$r_{e_{g}}^{\text {test }}$ & $0.2236(0.5247)$ & $0.1729(0.3532)$ & $0.0817(0.3587)$ & $\mathbf{0 . 0 0 2 7}(\mathbf{0 . 5 3 6 4})$ & $0.0029(0.5309)$ & $0.0029(0.4907)$ \\
$r_{e_{p}}^{\text {test }}$ & $0.5432(0.3757)$ & $0.3730(0.5249)$ & $0.1360(0.2709)$ & $\mathbf{0 . 0 0 2 8}(\mathbf{0 . 4 5 1 5})$ & $0.0031(0.2166)$ & $0.0033(0.4749)$ \\
$c_{e_{g}}^{\text {test }}$ & $0.2733(0.2104)$ & $0.1731(0.3326)$ & $0.0829(0.3310)$ & $\mathbf{0 . 0 0 2 7}(\mathbf{0 . 4 9 9 7})$ & $0.0029(0.3874)$ & $0.0029(0.4969)$ \\
$r_{e_{g}}^{\text {stest }}$ & $0.2830(0.3424)$ & $0.1632(0.4303)$ & $0.0932(0.5882)$ & $\mathbf{0 . 0 0 2 9}(\mathbf{0 . 4 9 2 7})$ & $\mathbf{0 . 0 0 2 9}(\mathbf{0 . 4 9 1 4})$ & $0.0031(0.5735)$ \\
$r_{e_{p}}^{\text {stest }}$ & $0.5729(0.5996)$ & $0.3829(0.5110)$ & $0.2532(0.5348)$ & $0.0032(0.4090)$ & $\mathbf{0 . 0 0 2 6}(\mathbf{0 . 4 5 8 2})$ & $0.0031(0.3844)$ \\
$c_{e_{g}}^{\text {stest }}$ & $0.2931(0.2220)$ & $0.1531(0.2105)$ & $0.0866(0.3779)$ & $\mathbf{0 . 0 0 2 6}(\mathbf{0 . 2 6 1 2})$ & $0.0030(0.4546)$ & $0.0028(0.5995)$ \\
$r_{e_{g}}^{\text {lest }}$ & $0.2429(0.2612)$ & $0.1733(0.2488)$ & $0.0832(0.5526)$ & $\mathbf{0 . 0 0 2 4}(\mathbf{0 . 2 5 2 1})$ & $0.0028(0.4023)$ & $0.0033(0.2909)$ \\
$r_{e_{p}}^{\text {tist }}$ & $0.6031(0.2359)$ & $0.4029(0.3856)$ & $0.2772(0.3256)$ & $\mathbf{0 . 0 0 2 5}(\mathbf{0 . 5 4 2 3})$ & $0.0031(0.5677)$ & $0.0030(0.2734)$ \\
$c_{e_{g}}^{\text {ltest }}$ & $0.2329(0.2745)$ & $0.1631(0.4322)$ & $0.0830(0.3358)$ & $0.0031(0.4016)$ & $\mathbf{0 . 0 0 2 7}(\mathbf{0 . 2 1 1 8})$ & $0.0031(0.4080)$ \\
\hline
\end{tabular}


TABLE 11: Compared machine learning algorithms in real data studies.

\begin{tabular}{|c|c|c|}
\hline Name & Description & Parameters \\
\hline SVMs & Standard single support vector machines with RBF kernel & $\begin{array}{l}\gamma_{\text {best }} \text { is obtained by grid searching in } \\
2^{[-20: 20]}\end{array}$ \\
\hline Ada-DT & AdaBoost using decision tree as weak classifiers & \\
\hline Ada-LSVMs & AdaBoost using linear kernel SVMs as weak classifiers & \\
\hline Ada-RSVMs & AdaBoost using RBF kernel SVMs as weak classifiers & $\begin{array}{l}\gamma_{\text {best }} \text { is obtained by grid searching in } \\
\qquad 2^{[-20: 20]}\end{array}$ \\
\hline RVM & Standard single relevance vector machines with RBF kernel & $\begin{array}{l}\gamma_{\text {best }} \text { is obtained by grid searching in } \\
\qquad 2^{[-20: 20]}\end{array}$ \\
\hline PRVM & $\begin{array}{c}\text { Relevance vector machines under the probit link function with RBF } \\
\text { kernel }\end{array}$ & $\begin{array}{l}\gamma_{\text {best }} \text { is obtained by grid searching in } \\
\qquad 2^{[-20: 20]}\end{array}$ \\
\hline ICET & $\begin{array}{l}\text { Incorporates misclassification costs in the fitness function of genetic } \\
\text { algorithms }\end{array}$ & \\
\hline $\begin{array}{l}\text { Cost-sensitive decision } \\
\text { trees }\end{array}$ & Use the misclassification costs in tree building process & \\
\hline MetaCost & $\begin{array}{l}\text { Convert cost-insensitive classifiers into cost-sensitive ones without } \\
\text { modifying }\end{array}$ & \\
\hline $\begin{array}{l}\text { Cost-sensitive naive } \\
\text { Bayes }\end{array}$ & Use cost factor information as the prior & \\
\hline Empirical thresholding & Use bagging on decision trees to obtain reliable probability estimations & \\
\hline Costing & Use rejection sampling to resample the data & \\
\hline Weighting & $\begin{array}{l}\text { Assign a normalized weight to each instance according to the } \\
\text { misclassification costs }\end{array}$ & \\
\hline
\end{tabular}

TABle 12: The results of seismic-bumps data $(b=15.13)$.

\begin{tabular}{|c|c|c|c|c|c|}
\hline \multirow{2}{*}{ Algorithms } & \multicolumn{5}{|c|}{ Evaluation measures } \\
\hline & Sensitivity & Specificity & F-1 Measure & AUC & Global Accuracy \\
\hline SVMs & 0.0000 & 1.0000 & 0.0000 & 0.5 & 0.9320 \\
\hline Ada-DT & 0.1047 & 0.9873 & 0.1636 & 0.5460 & 0.9273 \\
\hline Ada-LSVMs & 0.0000 & 0.9992 & 0.0000 & 0.4996 & 0.9312 \\
\hline Ada-RSVMs & 0.6279 & 0.6675 & 0.2030 & 0.6477 & 0.6648 \\
\hline RVM & 0.5318 & 0.9755 & 0.8601 & 0.2101 & 0.6533 \\
\hline PRMV & 0.9011 & 0.9364 & 0.9601 & 0.9101 & 0.9473 \\
\hline ICET & 0.9113 & 0.9578 & 0.9221 & 0.9122 & 0.9361 \\
\hline Cost-sensitive decision trees & 0.8804 & 0.9382 & 0.9130 & 0.9143 & 0.9473 \\
\hline MetaCost & 0.9043 & 0.9461 & 0.9316 & 0.9006 & 0.9422 \\
\hline Cost-sensitive naive Bayes & 0.9018 & 0.9418 & 0.9088 & 0.9200 & 0.9461 \\
\hline Empirical thresholding & 0.8933 & 0.9655 & 0.9287 & 0.9155 & 0.9327 \\
\hline Costing & 0.9161 & 0.9285 & 0.9129 & 0.9267 & 0.9378 \\
\hline Weighting & 0.9065 & 0.9499 & 0.9016 & 0.9331 & 0.9502 \\
\hline AdaImC & 0.9277 & 0.9853 & 0.9112 & 0.9376 & 0.9588 \\
\hline
\end{tabular}

better than other algorithms. The AdaImC obviously outperforms the PRVM except for the $F-1$ Measure.

\subsubsection{Glass-2 Data Study. The Glass-2 dataset is obtained} from the Knowledge Extraction based on Evolutionary Learning (KEEL) Dataset Repository [50]. This dataset is originally from the US Forensic Science Service and intensely imbalanced with only 17 samples of the positive class among a total of 214 samples, which means $b=11.59$. The Glass- 2 dataset has eight explanatory variables, including the refractive index and the different chemical elements content. The positive class is labeled as the glass made by the nonfloat process and indicated by $y=1$. The negative class is defined as the glass made by other processed methods and indicated by $y=-1$.
Table 13 displays the Glass-2 data experiment. It shows similar results as the seismic-bumps data. Except for the Specificity, all evaluation measures of PRVM and AdaImC are better than other algorithms. Compared to the PRVM, our proposed AdaImC has a slight improvement.

5.2.3. New-Thyroid-1 Data Study. The new-thyroid-1 dataset is also obtained from the KEEL Dataset Repository. This dataset is originally from the Garvan Institute in Sydney, Australia. It includes the information of thyroid patients and is rearranged by KEEL to be an imbalanced binary dataset. The new-thyroid-1 dataset is imbalanced with 35 positive samples among a total of 215 samples, which indicates the imbalanced index is $b=5.14$. This dataset contains 5 explanatory variables, including the levels of 
TABLE 13: The results of Glass-2 data $(b=11.59)$.

\begin{tabular}{|c|c|c|c|c|c|}
\hline \multirow{2}{*}{ Algorithms } & \multicolumn{5}{|c|}{ Evaluation measures } \\
\hline & Sensitivity & Specificity & F-1 Measure & AUC & Global Accuracy \\
\hline SVMs & 0.0000 & 1.0000 & 0.0000 & 0.5 & 0.8972 \\
\hline Ada-DT & 0.0909 & 0.9896 & 0.1538 & 0.5402 & 0.8972 \\
\hline Ada-LSVMs & 0.0000 & 1.0000 & 0.0000 & 0.5000 & 0.8970 \\
\hline Ada-RSVMs & 0.1818 & 0.9063 & 0.1818 & 0.5440 & 0.8318 \\
\hline RVM & 0.1433 & 0.9588 & 0.1710 & 0.6031 & 0.8313 \\
\hline PRMV & 0.9267 & 0.9088 & 0.9433 & 0.9276 & 0.9006 \\
\hline ICET & 0.9133 & 0.9526 & 0.9376 & 0.9273 & 0.9361 \\
\hline Cost-sensitive decision trees & 0.9063 & 0.9727 & 0.9288 & 0.9188 & 0.9422 \\
\hline MetaCost & 0.9122 & 0.9528 & 0.9476 & 0.9381 & 0.9418 \\
\hline Cost-sensitive naive Bayes & 0.9005 & 0.9317 & 0.9702 & 0.9427 & 0.9355 \\
\hline Empirical thresholding & 0.8973 & 0.9466 & 0.9466 & 0.9329 & 0.9341 \\
\hline Costing & 0.9121 & 0.9752 & 0.9310 & 0.9404 & 0.9362 \\
\hline Weighting & 0.9033 & 0.9499 & 0.9341 & 0.9387 & 0.9132 \\
\hline AdaImC & 0.9293 & 0.9019 & 0.9518 & 0.9786 & 0.9470 \\
\hline
\end{tabular}

TABLe 14: The results of new-thyroid-1 data $(b=5.14)$.

\begin{tabular}{|c|c|c|c|c|c|}
\hline \multirow{2}{*}{ Algorithms } & \multicolumn{5}{|c|}{ Evaluation measures } \\
\hline & Sensitivity & Specificity & F-1 Measure & AUC & Global Accuracy \\
\hline SVMs & 0.2632 & 1.0000 & 0.4167 & 0.6316 & 0.8704 \\
\hline Ada-DT & 0.7895 & 0.9888 & 0.8571 & 0.8891 & 0.9537 \\
\hline Ada-LSVMs & 0.8421 & 0.9888 & 0.8889 & 0.9154 & 0.9630 \\
\hline Ada-RSVMs & 0.8421 & 0.9663 & 0.8421 & 0.9042 & 0.9444 \\
\hline RVM & 0.8311 & 0.9472 & 0.8732 & 0.9031 & 0.9135 \\
\hline PRMV & 0.9133 & 0.9846 & 0.9572 & 0.9731 & 0.9655 \\
\hline ICET & 0.9172 & 0.9679 & 0.9221 & 0.9122 & 0.9361 \\
\hline Cost-sensitive decision trees & 0.8954 & 0.9755 & 0.9130 & 0.9143 & 0.9473 \\
\hline MetaCost & 0.9007 & 0.9421 & 0.9316 & 0.9006 & 0.9422 \\
\hline Cost-sensitive naive Bayes & 0.9110 & 0.9049 & 0.9088 & 0.9200 & 0.9461 \\
\hline Empirical thresholding & 0.8894 & 0.9482 & 0.9287 & 0.9155 & 0.9327 \\
\hline Costing & 0.9161 & 0.9562 & 0.9331 & 0.9267 & 0.9378 \\
\hline Weighting & 0.9048 & 0.9573 & 0.9016 & 0.9331 & 0.9502 \\
\hline AdaImC & 0.9148 & 0.9833 & 0.9620 & 0.9744 & 0.9670 \\
\hline
\end{tabular}

different hormones such as thyroxin and triiodothyronine. The positive class is labeled as hyperthyroidism, which is indicated by $y=1$. The negative class is defined as nonhyperthyroidism, which is indicated by $y=-1$.

Table 14 displays the results of the new-thyroid-1 data experiment. It shows the satisfactory performance of our proposed algorithm compared to others for this data classification problem. Almost all the evaluation criteria of the PRVM and the AdaImC perform better than other algorithms. Because $b=5.14$ is a relatively small number in this dataset, the differences between PRVM and AdaImC are not significant.

5.2.4. Ecoli-1 Data Study. The Ecoli-1 dataset is also obtained from the KEEL Dataset Repository. This dataset initially comes from the Institute of Molecular and Cellular Biology at Osaka University. It includes the information of the cellular localization sites of proteins and is rearranged by KEEL as an imbalanced binary dataset. The Ecoli-1 dataset is imbalanced with 77 positive samples among a total of 336 samples, which indicates the imbalanced index is $b=3.36$.
This dataset contains seven explanatory variables, including the signal sequence recognition scores by different methods and the presence of charge on N-terminus. The positive class is labeled as the inner membrane without a signal sequence, which is indicated by $y=1$. The negative class is defined as other situations, which is indicated by $y=-1$.

Table 15 displays the results of the Ecoli-1 data experiment. It shows all the algorithms perform similarly since the imbalanced situation is not obvious. Our proposed AdaImC still has a convincing performance for this balanced dataset.

Our proposed AdaImC algorithm outperforms the traditional ones in imbalanced data classification based on real data studies. With a large imbalanced index $b$, our proposed algorithm shows excellent performance.

5.3. Algorithm Complexity Analysis. Our proposed AdaImC algorithm outperforms the classical AdaBoost, especially in the imbalanced data problem. However, it has a more complex structure, which means the model complexity is larger than the classic one. In practice, especially for big data, our proposed one may face an inefficiency issue. 
TABle 15: The results of Ecoli-1 data $(b=3.36)$.

\begin{tabular}{|c|c|c|c|c|c|}
\hline \multirow{2}{*}{ Algorithms } & \multicolumn{5}{|c|}{ Evaluation measures } \\
\hline & Sensitivity & Specificity & F-1 Measure & AUC & Global Accuracy \\
\hline SVMs & 0.8889 & 0.9242 & 0.8205 & 0.9066 & 0.9167 \\
\hline Ada-DT & 0.8611 & 0.9091 & 0.7848 & 0.8851 & 0.8988 \\
\hline Ada-LSVMs & 0.0000 & 1.0000 & 0.0000 & 0.5000 & 0.7857 \\
\hline Ada-RSVMs & 0.9444 & 0.8636 & 0.7727 & 0.9040 & 0.8810 \\
\hline RVM & 0.9531 & 0.9143 & 0.8331 & 0.9212 & 0.9145 \\
\hline PRMV & 0.9779 & 0.9431 & 0.9822 & 0.9221 & 0.9055 \\
\hline ICET & 0.9466 & 0.9588 & 0.9642 & 0.9067 & 0.8730 \\
\hline Cost-sensitive decision trees & 0.9512 & 0.9831 & 0.9580 & 0.9122 & 0.8933 \\
\hline MetaCost & 0.9488 & 0.9793 & 0.9731 & 0.9052 & 0.8517 \\
\hline Cost-sensitive naive Bayes & 0.9571 & 0.9683 & 0.9738 & 0.9180 & 0.7841 \\
\hline Empirical thresholding & 0.9370 & 0.9681 & 0.9639 & 0.9033 & 0.8905 \\
\hline Costing & 0.9519 & 0.9488 & 0.9488 & 0.9073 & 0.9022 \\
\hline Weighting & 0.9611 & 0.9769 & 0.9581 & 0.9180 & 0.8467 \\
\hline AdaImC & 0.9644 & 0.9355 & 0.9883 & 0.9275 & 0.9108 \\
\hline
\end{tabular}

TABLE 16: The results of algorithms' running Time ${ }^{a}$.

\begin{tabular}{lcccc}
\hline \multirow{2}{*}{ Algorithms } & & \multicolumn{2}{c}{ Code running time (seconds) } & \\
& Seismic-bumps & Glass-2 & New-thyroid-1 & Ecoli-1 \\
\hline AdaBoost & 54.33 & 36.12 & 29.80 & 27.00 \\
AdaImC & 61.73 & 40.51 & 31.22 & 32.91 \\
Increase percentage & $13.62 \%$ & $12.15 \%$ & $4.77 \%$ & $21.89 \%$ \\
\hline
\end{tabular}

${ }^{a}$ Performed in Python 3.6 on macOS 10.12.6.

Assume that $c_{1}$ has been determined. Compared with the classic AdaBoost (Algorithm 1), the AdaImC (Algorithm 4) has the following additional computation for the training dataset S:

Step 0: " $n+2 n$," where " $n$ " is the calculated amount for $D_{t}(i)=c_{1}^{\left(1-y_{i}\right) / 2}$ and " $2 n$ " is the calculated amount for the

$$
0<c_{2}<\frac{1}{2} \ln \left(\frac{\sum_{i: h_{t}\left(\mathbf{x}_{i}\right)=y_{i}} D_{i}^{t} c_{1}^{\left(1-y_{i}\right) / 2}}{\sum_{i: h_{t}\left(\mathbf{x}_{i}\right) \neq y_{i}} D_{i}^{t} c_{1}^{\left(1-y_{i}\right) / 2}}\right) .
$$

Step 2: " $2 n$ " is the calculated amount for

$$
\alpha_{t}=\frac{1}{2} \ln \left(\frac{\sum_{i: h_{t}\left(\mathbf{x}_{i}\right)=y_{i}} D_{i}^{t} c_{1}^{\left(1-y_{i}\right) / 2}}{\sum_{i: h_{t}\left(\mathbf{x}_{i}\right) \neq y_{i}} D_{i}^{t} c_{1}^{\left(1-y_{i}\right) / 2}}\right)-c_{2} .
$$

Step 3: " $3 n$ " is the calculated amount for

$$
D_{t+1}^{i}=\frac{c_{1}^{\left(1-y_{i}\right) / 2} D_{t}^{i} \exp \left[-y_{i} h_{t}\left(\mathbf{x}_{i}\right)\left(\alpha_{t}+c_{2}\right)\right]}{n \prod_{t=1}^{T} \mathbf{Z}_{t}} .
$$

Totally, AdaImC has the additional $8 n$ computations than the classic AdaBoost, which seems acceptable for the practice. We compare the code running time for AdaImC and the classic AdaBoost based on the above four real datasets in Table 16. In relatively big datasets, the AdaImC needs significantly more time in practice. The additional time for code running is acceptable, and the complexity does not lower the usability of our proposed algorithm based on this limited study.

\section{Conclusion and Discussion}

The cost-sensitive learning model and the AdaBoost algorithm are two main approaches in imbalanced data classification. The combination of them, namely, cost-sensitive AdaBoost, is receiving more attention. Nevertheless, the challenging problem is that almost all cost-sensitive boosting algorithms have no theoretic proof, although they perform nicely in practice. The present article tries to solve this problem by studying the cost-sensitive AdaBoost algorithms for the imbalanced data classification with two contributions. First, we summarize most cost-sensitive AdaBoost algorithms in the literature, which are all heuristic models without theoretic proof. A comprehensively general form is proposed, and we set a particular one, namely, the AdaImC algorithm. Our proposed model considers adjusting the weight parameter of weak classifiers and the weight parameter of the data samples in the AdaBoost algorithm with the cost factors. A theoretic proof of boosting property is given to support our algorithm. Second, we study the PoE (Product of Experts) model in statistics. We conclude that our proposed AdaImC algorithm is reasonable since a particular form of the PoE model is identical to the AdaImC algorithm. Besides, the PoE model provides a deeper insight into the relationship between the cost parameters, which helps us determine the values of cost factors more logically.

More studies for cost-sensitive AdaBoost are ponderable in the future. The spontaneous idea extends to the multiple classes' problem, which has a more significant practical 
effect. The present article only considers a particular form for the cost-sensitive AdaBoost, but we can focus on the general form and provide the theoretical proofs from machine learning and statistics perspectives. Also, the choices of the values of the cost parameters are limited in this paper. A broader range of the values of parameters would provide more convincing results. Another point worth mentioning is that the proposed model is not robust under seriously imbalanced datasets with imbalanced index larger than 20, where the outlier detection algorithms are more suitable.

\section{Appendix}

\section{A. Proof of Theorem 2}

Proof

$$
D_{t+1}^{i}=\frac{c_{1}^{\left(1-y_{i}\right) / 2} \exp \left[-y_{i} h_{t}\left(\mathbf{x}_{i}\right)\left(\alpha_{t}+c_{2}\right)\right]}{n \prod_{t=1}^{T} \mathbf{Z}_{t}},
$$

where $c_{1}$ and $c_{2}$ are constant numbers, $0<c_{1}<1$, and $c_{2}>0$. We have $\left|h\left(\mathbf{x}_{i}\right) \neq y_{i}\right| \leq \exp \left[-y_{i} h_{t}\left(\mathbf{x}_{i}\right)\left(\alpha_{t}+c_{2}\right)\right]$ and

$$
\begin{aligned}
\frac{1}{n} \sum_{i=1}^{n}\left|h\left(\mathbf{x}_{i}\right) \neq y_{i}\right| & \leq \frac{1}{n} \sum_{i=1}^{n} \exp \left[-y_{i} h_{t}\left(\mathbf{x}_{i}\right)\left(\alpha_{t}+c_{2}\right)\right] \\
& =\sum_{i=1}^{n}\left(\prod_{t=1}^{T} \mathbf{Z}_{t}\right) D_{t+1}^{i} / c_{1}^{\left(1-y_{i}\right) / 2} \\
& \leq \sum_{i=1}^{n}\left(\prod_{t=1}^{T} \mathbf{Z}_{t}\right) D_{t+1}^{i} / c_{1} \\
& =\frac{1}{c_{1}} \prod_{t=1}^{T} \mathbf{Z}_{t} .
\end{aligned}
$$

\section{B. Proof of Theorem 3}

Proof. When $h_{t}\left(\mathbf{x}_{i}\right) \in\{1,1\}$, which means $y_{i} h_{t}\left(\mathbf{x}_{i}\right) \in$ $\{-1,1\}$,

$$
\begin{aligned}
& \mathbf{Z}_{t}=\sum_{i=1}^{n} D_{t}^{i} c_{1}^{\left(1-y_{i}\right) / 2} \exp \left[-y_{i} h_{t}\left(\mathbf{x}_{i}\right)\left(\alpha_{t}+c_{2}\right)\right] \\
& \leq \sum_{i=1}^{n} D_{t}^{i} c_{1}^{\left(1-y_{i}\right) / 2}\left(\frac{1+y_{i} h_{t}\left(\mathbf{x}_{i}\right)}{2} \exp \left(-\alpha_{t}-c_{2}\right)\right. \\
& \left.+\frac{1-y_{i} h_{t}\left(\mathbf{x}_{i}\right)}{2} \exp \left(\alpha_{t}+c_{2}\right)\right) \\
& \alpha_{t}=\arg \min \sum_{i=1}^{n} D_{t}^{i} c_{1}^{\left(1-y_{i}\right) / 2}\left(\frac{1+y_{i} h_{t}\left(\mathbf{x}_{i}\right)}{2} .\right. \\
& \left.\exp \left(-\alpha_{t}-c_{2}\right)+\frac{1-y_{i} h_{t}\left(\mathbf{x}_{i}\right)}{2} \exp \left(\alpha_{t}+c_{2}\right)\right) \\
& =\frac{1}{2} \ln \left(\frac{\sum_{i: h_{t}\left(\mathbf{x}_{i}\right)=y_{i}} D_{i}^{t} c_{1}^{\left(1-y_{i}\right) / 2}}{\sum_{i: h_{t}\left(\mathbf{x}_{i}\right) \neq y_{i}} D_{i}^{t} c_{1}^{\left(1-y_{i}\right) / 2}}\right)-c_{2} .
\end{aligned}
$$

\section{Proof of Theorem 4}

Proof. Let

$$
\begin{aligned}
L_{j} & =\ln p\left(y_{1}, y_{2}, \ldots, y_{n} \mid \mathbf{x}_{1}, \mathbf{x}_{2}, \ldots, \mathbf{x}_{n}, h_{1}(\mathbf{x}), h_{2}(\mathbf{x}), \ldots, h_{j}(\mathbf{x})\right), \\
L_{j-1} & =\ln p\left(y_{1}, y_{2}, \ldots, y_{n} \mid \mathbf{x}_{1}, \mathbf{x}_{2}, \ldots, \mathbf{x}_{n}, h_{1}(\mathbf{x}), h_{2}(\mathbf{x}), \ldots, h_{j-1}(\mathbf{x})\right) .
\end{aligned}
$$


The requirement of $L_{j} \geq L_{j-1}$ results in

$$
\sum_{i=1}^{n} \ln \left(Q_{j-1}^{i}+\frac{p\left(\overline{y_{i}} \mid \mathbf{x}_{i}, h_{j}\right)}{p\left(y_{i} \mid \mathbf{x}_{i}, h_{j}\right)} \bar{Q}_{j-1}^{i}\right) \leq 0,
$$

where $Q_{j-1}^{i}=p\left(y_{i} \mid \mathbf{x}_{i}, h_{1}, \ldots, h_{j-1}\right)$ and $\bar{Q}_{j-1}^{i}=p\left(\overline{y_{i}} \mid \mathbf{x}_{i}, h_{1}\right.$, $\left.\ldots, h_{j-1}\right)$. We cannot find closed form of solution for (28), and relax the upper bound using Jensen's inequality:

$$
\begin{aligned}
& \frac{1}{n} \sum_{i=1}^{n} \ln \left(Q_{j-1}^{i}+\frac{p\left(\overline{y_{i}} \mid \mathbf{x}_{i}, h_{j}\right)}{p\left(y_{i} \mid \mathbf{x}_{i}, h_{j}\right)} \bar{Q}_{j-1}^{i}\right) \leq \\
& \ln \frac{1}{n} \sum_{i=1}^{n}\left(Q_{j-1}^{i}+\frac{p\left(\overline{y_{i}} \mid \mathbf{x}_{i}, h_{j}\right)_{\bar{Q}_{j-1}}}{p\left(y_{i} \mid \mathbf{x}_{i}, h_{j}\right)}\right) \leq 0 .
\end{aligned}
$$

Simplifying (30), we obtain (27).

\section{Data Availability}

Four real binary datasets used to support the findings of this study have been deposited in the KEEL imbalanced data repository, https://sci2s.ugr.es/keel/imbalanced.php (Alberto Fernández, Salvador García, María José del Jesus, and Francisco Herrera, "A study of the behaviour of linguistic fuzzy rule based classification systems in the framework of imbalanced datasets," Fuzzy Sets and Systems, 159(18):2378-2398, 2008).

\section{Conflicts of Interest}

The authors declare that there are no conflicts of interest regarding the publication of this article.

\section{Acknowledgments}

This work was supported by the Key Project of National Natural Science Foundation of China (No. 71831002), 2021 Natural Science Foundation of Liaoning Province (No. 2021BS-076), National Key Research and Development Program of China (No. 2019YFB1600400), National Natural Science Foundation of China (No. 71774018), China Postdoctoral Science Foundation (No. 2015M580128), and Natural Science Foundation of Liaoning Province (No. 2020-HYLH-20).

\section{References}

[1] P. K. Chan and J. S. Salvatore, “Toward scalable learning with non-uniform class and cost distributions: a case study in credit card fraud detection," KDD, vol. 98, pp. 164-168, 1998.

[2] A. Onan, "Biomedical text categorization based on ensemble pruning and optimized topic modelling," Computational and Mathematical Methods in Medicine, vol. 2018, Article ID 2497471, 22 pages, 2018.

[3] A. Onan, S. Korukoğlu, and H. Bulut, "A multiobjective weighted voting ensemble classifier based on differential evolution algorithm for text sentiment classification," Expert Systems with Applications, vol. 62, pp. 1-16, 2016.

[4] A. Onan, "Classifier and feature set ensembles for web page classification," Journal of Information Science, vol. 42, no. 2, pp. 150-165, 2016.
[5] A. Onan, "A fuzzy-rough nearest neighbor classifier combined with consistency-based subset evaluation and instance selection for automated diagnosis of breast cancer," Expert Systems with Applications, vol. 42, no. 20, pp. 6844-6852, 2015.

[6] M. Kubat, R. C. Holte, and S. Matwin, "Machine learning for the detection of oil spills in satellite radar images," Machine Learning, vol. 30, no. 2, pp. 195-215, 1998.

[7] E. Alpaydin, Introduction to Machine Learning, MIT Press, Cambridge, MA, USA, 2009.

[8] Y. Sun, M. S. Kamel, A. K. C. Wong, and Y. Wang, "Costsensitive boosting for classification of imbalanced data," Pattern Recognition, vol. 40, no. 12, pp. 3358-3378, 2007.

[9] X. Li, L. Wang, and E. Sung, "AdaBoost with SVM-based component classifiers," Engineering Applications of Artificial Intelligence, vol. 21, no. 5, pp. 785-795, 2008.

[10] P. P. Sotiris Kotsiantis and D. Kanellopoulos, "Handling imbalanced datasets a review," GESTS Interuational Transactions on Computer Science and Engineering, vol. 30, 2006.

[11] G. M. Weiss, "Mining with rarity," ACM SIGKDD Explorations Newsletter, vol. 6, no. 1, pp. 7-19, 2004.

[12] X. Tao, Q. Li, W. Guo et al., "Adaptive weighted over-sampling for imbalanced datasets based on density peaks clustering with heuristic filtering," Information Sciences, vol. 519, pp. 43-73, 2020.

[13] X. Tao, Q. Li, W. Guo et al., "Self-adaptive cost weights-based support vector machine cost-sensitive ensemble for imbalanced data classification," Information Sciences, vol. 487, pp. 31-56, 2019.

[14] R. Chao, X. Tao, Q. Li et al., "Real-value negative selection over-sampling for imbalanced data set learning," Expert Systems with Applications, vol. 129, pp. 118-134, 2019.

[15] F. Cheng, J. Zhang, and C. Wen, "Cost-sensitive large margin distribution machine for classification of imbalanced data," Pattern Recognition Letters, vol. 80, pp. 107-112, 2016.

[16] P. D. Turney, "Low size-complexity inductive logic programming: the east-west challenge considered as a problem in cost-sensitive classification," 2002, https://arxiv.org/abs/cs/ 0212039.

[17] C. X. Ling and V. S. Sheng, "Cost-sensitive learning and the class imbalance problem," Encyclopedia of machine learning, vol. 2011, pp. 231-235, 2008.

[18] D. Pedro, "Metacost: a general method for making classifiers cost-sensitive," in Proceedings of the fifth ACM SIGKDD international conference on Knowledge discovery and data mining, pp. 155-164, ACM, San Diego, CA USA, August 1999.

[19] X. Chai, L. Deng, Q. Yang, and C. X. Ling, "Test-cost sensitive naive bayes classification," in Proceedings of the Fourth IEEE International Conference on Data Mining (ICDM'04), pp. 51-58, IEEE, Brighton, UK, November 2004.

[20] B. Zadrozny, J. Langford, and N. Abe, "Cost-sensitive learning by cost-proportionate example weighting," in Proceedings of the Third IEEE International Conference on Data Mining, 2003, pp. 435-442, IEEE, Melbourne, FL, USA, November 2003.

[21] K. M. Ting, "Inducing cost-sensitive trees via instance weighting," in Proceedings of the European Symposium On Principles Of Data Mining And Knowledge Discovery, pp. 139-147, Springer, Nantes, France, September 1998.

[22] M. V. Joshi, V. Kumar, and R. C. Agarwal, "Evaluating boosting algorithms to classify rare classes: comparison and improvements," in Proceedings of the IEEE International Conference on Data Mining, 2001, pp. 257-264, IEEE, San Jose, CA, USA, November 2001. 
[23] K. Grigoris and J. Shawe-Taylor, "Optimizing classifers for imbalanced training sets," in Proceedings of the Advances in Neural Information Processing Systems, pp. 253-259, Denver, CO, USA, December 1999.

[24] W. Wang and D. Sun, "The improved adaboost algorithms for imbalanced data classification," Information Sciences, vol. 563, pp. 358-374, 2021.

[25] M. Galar, A. Fernández, E. Barrenechea, H. Bustince, and F. Herrera, "Ordering-based pruning for improving the performance of ensembles of classifiers in the framework of imbalanced datasets," Information Sciences, vol. 354, pp. 178-196, 2016.

[26] R. E. Schapire, “The strength of weak learnability," Machine Learning, vol. 5, no. 2, pp. 197-227, 1990.

[27] Y. Freund and R. E. Schapire, "A decision-theoretic generalization of on-line learning and an application to boosting," Journal of Computer and System Sciences, vol. 55, no. 1, pp. 119-139, 1997.

[28] L. Chang and H. Rong, "Application of an improved bpadaboost model in semiconductor quality prediction," in Proceedings of the 2019 IEEE International Symposium on Predictive Control of Electrical Drives and Power Electronics (PRECEDE), Quanzhou, China, May 2019.

[29] R. E. Schapire and Y. Singer, "Improved boosting algorithms using confidence-rated predictions," Machine Learning, vol. 37, no. 3, pp. 297-336, 1999.

[30] Y. Freund, R. Schapire, and N. Abe, "A short introduction to boosting," Journal of Japanese Society for Artificial Intelligence, vol. 14, no. 771-780, p. 1612, 1999.

[31] C. Elkan, "The foundations of cost-sensitive learning,"vol. 17, pp. 973-978, in Proceedings of the International Joint Conference on Artificial Intelligence, vol. 17, pp. 973-978, Lawrence Erlbaum Associates Ltd, Barcelona, Spain, July 2001.

[32] H. Lu, H. Gao, M. Ye, Ke Yan, and X. Wang, "A hybrid ensemble algorithm combining adaboost and genetic algorithm for cancer classification with gene expression data," in Proceedings of the 2018 9th International Conference on Information Technology in Medicine and Education (ITME), Hangzhou, China, October 2018.

[33] S. Lomax and S. Vadera, "A survey of cost-sensitive decision tree induction algorithms," ACM Computing Surveys, vol. 45, no. 2, p. 16, 2013.

[34] V. López, A. Fernández, J. G. Moreno-Torres, and F. Herrera, "Analysis of preprocessing vs. cost-sensitive learning for imbalanced classification. open problems on intrinsic data characteristics," Expert Systems with Applications, vol. 39, no. 7, pp. 6585-6608, 2012.

[35] L. Breiman, Classification and Regression Trees, Routledge, England, UK, 2017.

[36] M. Kubat and S. Matwin, "Addressing the curse of imbalanced training sets: one-sided selection," Icml, vol. 97, pp. 179-186, 1997.

[37] P. D. Turney, "Types of cost in inductive concept learning," 2002, https://arxiv.org/abs/cs/0212034.

[38] W. Fan, J. Salvatore, J. Zhang, and P. K. Chan, "Adacost: misclassification cost-sensitive boosting," in Proceedings of the Icml, pp. 97-105, New York, NY, USA, June 1999.

[39] K. M. Ting, "A comparative study of cost-sensitive boosting algorithms," in Proceedings of the 17th International Conference on Machine Learning, Citeseer, San Francisco, CA, USA, June 2000.

[40] V. Paul and M. Jones, "Fast and robust classification using asymmetric adaboost and a detector cascade," in Proceedings of the Advances in Neural Information Processing Systems, pp. 1311-1318, Vancouver, Canada, December 2002.

[41] I. Landesa-Vázquez and J. L. Alba-Castro, "Shedding light on the asymmetric learning capability of adaboost," Pattern Recognition Letters, vol. 33, no. 3, pp. 247-255, 2012.

[42] H. Masnadi-Shirazi and N. Vasconcelos, "Asymmetric boosting," in Proceedings of the 24th international conference on Machine learning, pp. 609-619, ACM, Buenos Aires, Argentina, July 2007.

[43] N. Nikolaou, E. Narayanan, M. Kull, P. Flach, and G. Brown, "Cost-sensitive boosting algorithms: do we really need them?" Machine Learning, vol. 104, no. 2-3, pp. 359-384, 2016.

[44] D. Lewis and W. Gale, "Training text classifiers by uncertainty sampling," 1994, https://arxiv.org/abs/cmp-lg/9407020.

[45] G. E. Hinton, "Training products of experts by minimizing contrastive divergence," Neural Computation, vol. 14, no. 8, pp. 1771-1800, 2002.

[46] W. Jiang, "Process consistency for adaboost," Annals of Statistics, vol. 32, no. 1, pp. 13-29, 2004.

[47] L. Yi, "A note on margin-based loss functions in classification," Statistics \& Probability Letters, vol. 68, no. 1, pp. 73-82, 2004.

[48] W. Wang, D. Sun, S. Peng, H. Kuang, and C. Sui, "Fully bayesian analysis of relevance vector machine classification with probit link function for imbalanced data problem," IEEE Access, vol. 9, 2021.

[49] M. Sikora, "Application of rule induction algorithms for analysis of data collected by seismic hazard monitoring systems in coal mines," Archives of Mining Sciences, vol. 55, no. 1, pp. 91-114, 2010.

[50] J. Alcalá-Fdez, A. Fernández, J. Luengo et al., "Keel datamining software tool: data set repository, integration of algorithms and experimental analysis framework," Journal of Multiple-Valued Logic and Soft Computing, vol. 17, 2011. 